\title{
OPEN A small molecule high throughput screening platform to profile conformational properties of nascent, ribosome-bound proteins
}

\author{
Hideki Shishido ${ }^{1,3}$, Jae Seok Yoon ${ }^{1} \&$ William R. Skach ${ }^{2 \bowtie}$
}

Genetic mutations cause a wide spectrum of human disease by disrupting protein folding, both during and after synthesis. Transient de-novo folding intermediates therefore represent potential drug targets for pharmacological correction of protein folding disorders. Here we develop a FRET-based high-throughput screening (HTS) assay in 1,536-well format capable of identifying small molecules that interact with nascent polypeptides and correct genetic, cotranslational folding defects. Ribosome nascent chain complexes (RNCs) containing donor and acceptor fluorophores were isolated from cell free translation reactions, immobilized on Nickel-NTA/IDA beads, and imaged by high-content microscopy. Quantitative FRET measurements obtained from as little as 0.4 attomole of protein/bead enabled rapid assessment of conformational changes with a high degree of reproducibility. Using this assay, we performed a pilot screen of $\sim 50,000$ small molecules to identify compounds that interact with RNCs containing the first nucleotide-binding domain (NBD1) of the cystic fibrosis transmembrane conductance regulator (CFTR) harboring a disease-causing mutation (A455E). Screen results yielded 133 primary hits and 1 validated hit that normalized FRET values of the mutant nascent peptide. This system provides a scalable, tractable, structure-based discovery platform for screening small molecules that bind to or impact the folding of protein substrates that are not amenable to traditional biochemical analyses.

In cells, nascent proteins begin to acquire three dimensional tertiary structure cotranslationally in a complex cellular environment as the nascent polypeptide emerges from the ribosome exit tunnel ${ }^{1-5}$. De novo protein folding can be influenced by biosynthetic machinery ${ }^{3,4}$ including the presence of the adjacent ribosome ${ }^{2-4}$, translation elongation rate $^{2-4,6,7}$, interactions with cellular chaperones ${ }^{3,4,8,9}$, and molecular crowding ${ }^{10-12}$. Events that occur during synthesis can also influence folding efficiency as well as functional properties of the final structure ${ }^{13,14}$. When folding is disrupted either by inherited or acquired mutations or perturbations of the cellular environment, resultant misfolded proteins can cause a diverse array of clinical disorders through loss of function, gain of toxic function, or cellular mislocalization and degradation by the ubiquitin proteasome system (UPS) ${ }^{15-18}$.

Cystic fibrosis (CF) is a prototypical protein folding disorder caused by mutations in the Cystic Fibrosis Transmembrane conductance Regulator (CFTR), an ABC transporter containing two six-spanning transmembrane domains (TMD1 and TMD2), two cytosolic nucleotide binding domains (NBD1 and NBD2), and an unstructured regulatory domain (R). CFTR functions as a PKA-regulated and ATP-gated chloride channel in the apical membrane of epithelial tissues where it controls salt and water movement ${ }^{19}$. More than 1,700 genetic variants have been reported for CFTR (www.genet.sickkids.on.ca/cftr), and it is estimated that several hundred variants cause CF by disrupting CFTR folding through various mechanisms. For example, F508del, which eliminates a phenylalanine at residue 508, decreases thermal stability of NBD1 $1^{20,21}$ and destabilizes an intramolecular interface between NBD1 and the 4th intracellular loop of TMD2 ${ }^{21-23}$. NBD1 folding is particularly susceptible to disease causing mutations due to its complex folding pathway, in which cellular biosynthetic machinery is required to facilitate formation of a parallel 4 -stranded hydrophobic $\beta$-sheet within the core of the domain ${ }^{1,2,24}$. Recently, we

${ }^{1}$ CFFT Lab, Cystic Fibrosis Foundation, 44 Hartwell Ave, Lexington, MA 02421, USA. ${ }^{2}$ Cystic Fibrosis Foundation, 4550 Montgomery Ave., Suite 1100N, Bethesda, MD 20814, USA. ${ }^{3}$ Present address: Generate Biomedicines, Inc., 26 Landsdowne St, Cambridge, MA 02139, USA. ${ }^{\varpi}$ email: wskach@cff.org 
showed that certain mutations in NBD1 can also alter the cotranslational folding pathway and thereby influence CFTR trafficking and stability ${ }^{24}$.

High-throughput screening (HTS) strategies identifying novel pharmacological chaperones for protein misfolding disorders, have typically relied on cellular assays such as measurements of the cell surface expression or function of target proteins ${ }^{25-27}$. For CF, phenotypic cell-based HTS assays have been widely used to screen for corrector molecules ${ }^{28,29}$ based on CFTR function at the plasma membrane ${ }^{30,31}$ or the trafficking of mutant CFTR. Purified protein domains have also been used in thermal shift assays ${ }^{32,33}$ to identify molecules that bind NBD1 directly. To date, HTS efforts have largely focused on F508del and have identified numerous corrector molecules ${ }^{34}$. These extensive efforts to correct F508del CFTR folding in cells, using small molecules, have led to recent FDA approval of three combination drugs: Lumacaftor (VX-809) + Ivacaftor in 2015, Tezacaftor (VX-661) + Ivacaftor in 2018, and Elexacaftor (VX-445) + Tezacaftor + Ivacaftor in 201935-38. Despite these advances, however, not all CFTR missense mutations respond to existing modulator drugs ${ }^{39}$. In addition, compounds must be present during CFTR synthesis to maximally stimulate CFTR folding ${ }^{36,40,41}$. This has suggested that some CFTR correctors may act on one or more transient biosynthetic intermediates ${ }^{42}$ and that understanding cotranslational folding pathways may provide a potential novel approach for developing new treatment strategies ${ }^{24}$.

A powerful method to study transient biosynthetic folding intermediates utilizes stable, Ribosome-bound Nascent Chain complexes (RNCs) derived from in vitro-translated truncated RNA transcripts ${ }^{1,2,43,44}$. In this system it is possible to quantitatively insert a donor fluorophore, Cyan Fluorescent Protein (CFP) at the N-terminus via a chimeric fusion, and a small acceptor dye (7-nitrobenz-2-oxa-1,3-diazole) at an engineered stop (TAG) codon within NBD1 using a synthetic aminoacylated suppressor tRNA ( $\varepsilon N-7$-nitrobenz-2-oxa-1,3-diazol $(\varepsilon N B D)-\left[{ }^{14} \mathrm{C}\right]$ Lys-tRNA $\left.{ }^{\mathrm{amb}}\right)^{1,2,44}$. Ribosomes that readthrough the stop codon, incorporate the acceptor dye and are arrested at the end of the truncated transcript at a defined stage of synthesis. Resultant polypeptides remain stably bound to the ribosome via a covalent peptidyl-tRNA bond (CFP-NBD1 RNCs), and are thus captured in the context of native biosynthetic machinery ${ }^{44}$. By adjusting translation conditions appropriately, donor and acceptor probes can be incorporated in near 1:1 stoichiometry, and RNC concentration can be precisely determined by incorporating a $\left[{ }^{14} \mathrm{C}\right]$ isotope into polypeptides during readthrough of the stop-codon (see "Methods"). It is therefore possible to simultaneously quantify nascent chain concentration and fluorescence intensity of donor and acceptor probes to determine Fluorescence Resonance Energy Transfer (FRET) at each truncation site as the nascent polypeptide transitions from an elongated, unfolded conformation (low FRET) to a more compact, folded

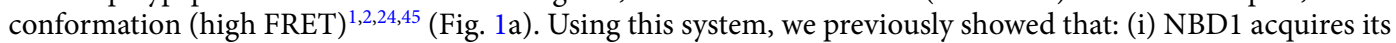
structure cotranslationally through a distinct series of carefully choreographed folding events, (ii) CF-causing mutations located within NBD1 can disrupt the nascent polypeptide folding landscape, and (iii) genetic suppressor mutations that restore cotranslational folding can partially restore trafficking of full-length CFTR ${ }^{1,2,24}$. These findings suggest that nascent folding intermediates may play an important role in disease pathogenesis and thus provide potential targets for pharmacological correction.

While cell-free translation allows one to precisely control both nascent chain length and site of probe incorporation, FRET analysis is technically challenging due to the low concentration of RNCs $(\sim 1-5 \mathrm{nM})$ that can be isolated from in vitro translation reactions. We therefore employed a strategy to immobilize RNCs to increase the effective local concentration and enable quantitative fluorescence measurements via high-content microscopic imaging (Fig. 1b). This approach dramatically increases sensitivity, enabling us to detect approximately 0.4 attomole of RNCs per bead $\left(2.4 \times 10^{5}\right.$ molecules $)$, and allows $\sim 20,000$ FRET measurements to be made from a single scaled translation reaction. By implementing a series of technical refinements, we were able to establish this system for HTS in 1,536-well plate format with high degree of reproducibility, and performed a pilot screen using a 50,000 small molecule compound library.

\section{Results}

Optimizing RNC binding and fluorescence detection. To improve sensitivity and scalability of fluorescence measurements, we systematically tested three immobilization strategies (streptavidin-biotin, $\mathrm{His}_{10}-\mathrm{Ni}-$ NTA/IDA, and myc (9E10) epitope antibody binding) using 12 commercially available solid supports (Supplementary Table S1). Ni-NTA/IDA binding was deemed most efficient, and three supports were identified with favorable binding efficiency, specificity, and optical characteristics: High Density Nickel 4 Highly Cross-linked Superfine $17 \mu \mathrm{m}$ (17 $\mu \mathrm{m}$ beads), HiTrap Chelating HP charged with Nickel (34 $\mu \mathrm{m}$ beads), and Ni-NTA agarose (100 $\mu \mathrm{m}$ beads). Imaging was initially performed on an inverted Olympus IX71 and a Nikon Ti-E eclipse microscope, and subsequently adapted to a GE IN Cell Analyzer 2200 high content imaging system because of its superior performance and high throughput capabilities. Bead binding characteristics were optimized using His $_{10^{-}}$-tagged and non-His-tagged CFP-NBD1 RNCs incubated with $2 \times 10^{5} 17 \mu \mathrm{m}$ beads, $5 \times 10^{4} 34 \mu \mathrm{m}$ beads, or $6 \times 10^{3} 100 \mu \mathrm{m}$ beads which provide similar aggregate binding surface area. All beads yielded satisfactory sixfold signal to background ratio (Fig. 1c-d). Net fluorescence signal was determined after flat field correction and subtraction of background (buffer) and nonspecific bead fluorescence (non-his-tagged constructs) (Fig. 1e). Net signal to noise (compared to non-His-tagged RNCs) was 103, 13, and 7 for $17 \mu \mathrm{m}, 34 \mu \mathrm{m}$, and $100 \mu \mathrm{m}$ beads, respectively. We also found that materials exhibited differences in variability in bead-to-bead fluorescence intensity, which impacted the reproducibility of individual FRET measurements. $17 \mu \mathrm{m}$ beads were superior due to less variation in bead measurements and nonspecific binding compared to $34 \mu \mathrm{m}$ and $100 \mu \mathrm{m}$ beads (Fig. 1e and Supplementary Fig. S1). For these reasons, $17 \mu \mathrm{m}$ beads (isolated within a narrow size range-16 $\mu \mathrm{m}$ to $23 \mu \mathrm{m}-$ by gravity segmentation) were selected among the three Ni-NTA/IDA supports for further study.

RNC bead capture efficiency was evaluated by varying bead number and binding time (Fig. 2). Incubation times were chosen based on binding kinetics, which were much slower for RNCs than free polypeptides due to spatial and rotational constraints imposed by the attached ribosome (Fig. 2a,b). Total binding (fluorescent signal/ 
a

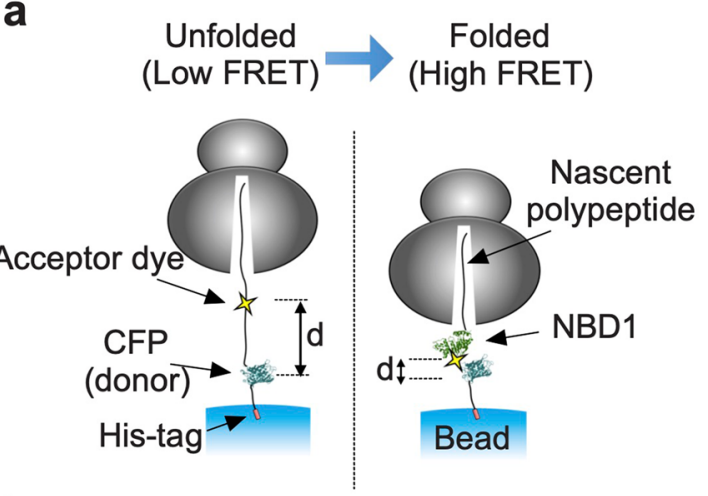

b

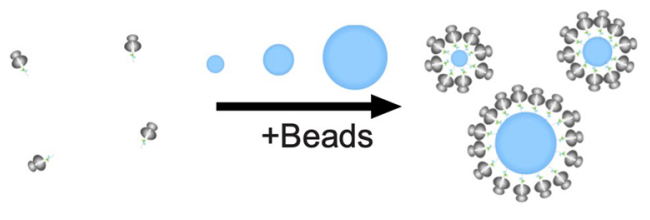

d

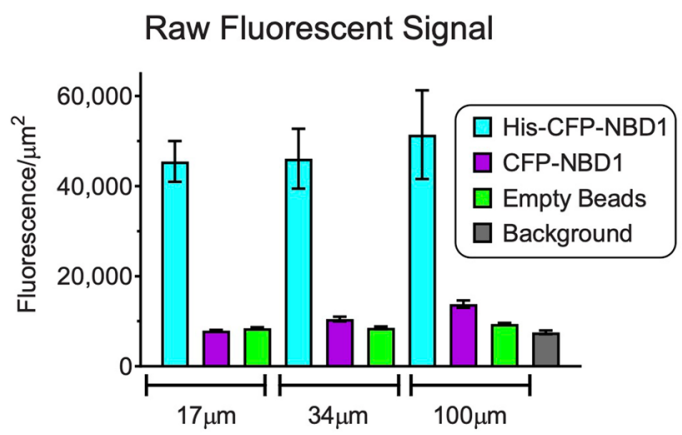

C

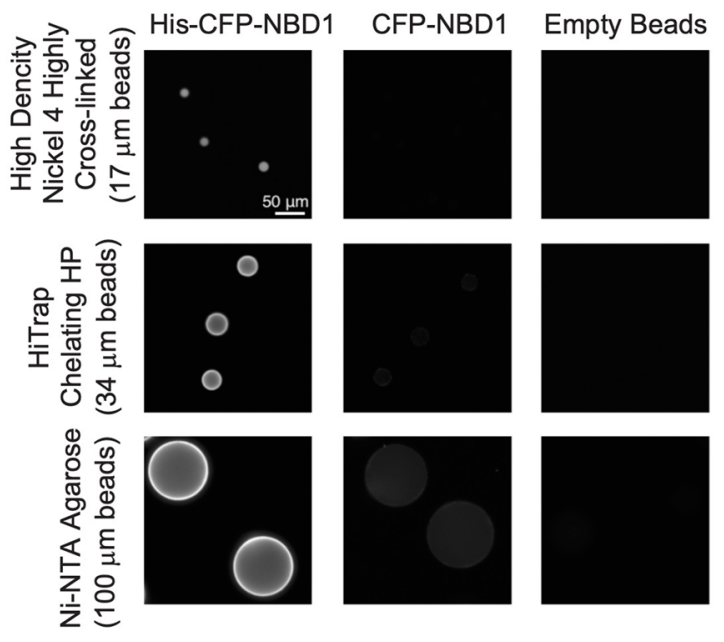

e

Net Fluorescent Signal

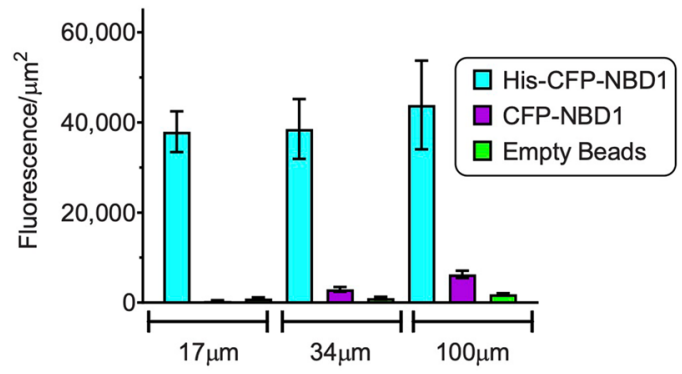

Figure 1. Fluorescent detection of immobilized RNCs. (a) Cartoon depicting solid-support FRET assay using translationally incorporated fluorophores to detect structural transitions of ribosome-attached nascent polypeptides immobilized on solid-support surface via His-tag. (b) Schematic showing increase in RNC concentration on surface of beads. (c) Raw images of beads containing RNC with $\mathrm{His}_{10}$-CFP-NBD1. Equivalent amount of RNCs were incubated with $2 \times 10^{5}$ of $17 \mu \mathrm{m}$ beads, $5 \times 10^{4}$ of $34 \mu \mathrm{m}$ beads, and $6 \times 10^{3}$ of $100 \mu \mathrm{m}$ beads as described in "Methods" section. Scale bar, $50 \mu \mathrm{m}$. (d) Mean fluorescence intensity $/ \mu \mathrm{m}^{2}$ was obtained from images (mean $\pm S D, n=12-99$ beads). Mean fluorescence intensities of beads containing His-CFP-NBD1 RNCs and background signal intensity (buffer) were used to calculate signal to background ratios. (e) Net fluorescence signal intensity $/ \mu \mathrm{m}^{2}$ was determined by subtracting background (buffer) (mean $\pm \mathrm{SD}$ ).

$\mu \mathrm{m}^{2}$ ) was inversely proportional to bead number and increased by both RNC concentration and binding time (Fig. $2 c, f$ ). RNC surface density was calculated based on the amount of $\left[{ }^{14} \mathrm{C}\right]$ Lys-labeled nascent polypeptide bound per total surface area of beads using average bead diameter estimates (Fig. 2d,g). Surface density correlated well with fluorescence signal intensity. Bead capture efficiency (RNCs bound/RNCs in binding reaction) was time dependent but relatively unaffected by RNC concentration (Fig. 2e,h). Importantly, some bead binding conditions are outliers, which may be indications of inappropriate binding conditions. $34 \mu \mathrm{m}$, and $100 \mu \mathrm{m}$ beads showed patterns similar to those of $17 \mu \mathrm{m}$ beads (Supplementary Fig. S2, S3). However larger beads resulted in fewer usable measurements and greater variation in fluorescence intensity. From these results, $2 \times 10^{5} 17 \mu \mathrm{m}$ beads, $400 \mu \mathrm{l}$ of $2 \mathrm{nM} \mathrm{RNC}$ and $\geq 6 \mathrm{~h}$ incubation time were chosen for further experiments as parameters that gave reasonable yield to use for HTS.

Solid-support FRET using immobilized RNCs. To determine whether substrate immobilization impacted nascent chain conformation, we compared steady state fluorescence measurements of RNCs in solution to measurements obtained from RNCs immobilized on bead surface. In each case, parallel, matched translation samples were used containing equal concentrations of nascent polypeptides that contained donor only (D), or donor + acceptor $(\mathrm{D}+\mathrm{A})$ fluorescent probes. For these experiments, the acceptor dye was incorporated at the CFP fusion site (residue 389 of CFTR) because FRET at this site depends only on the short tether distance to the CFP chromophore ${ }^{1}$ (Fig. 3a). FRET measurements were calculated as reported previously based on the decrease in donor fluorescence intensity due to the presence of the acceptor probe using the formula: 
a

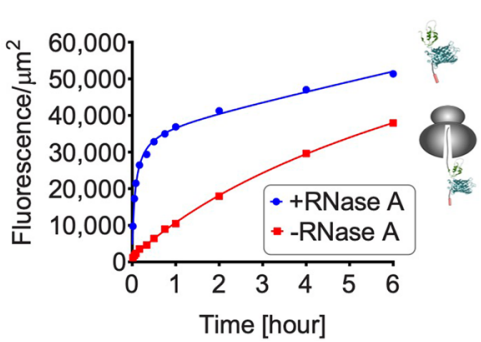

c

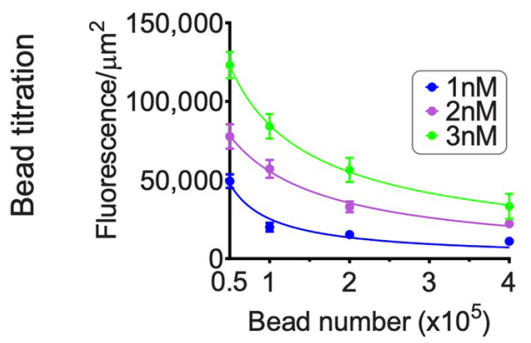

f

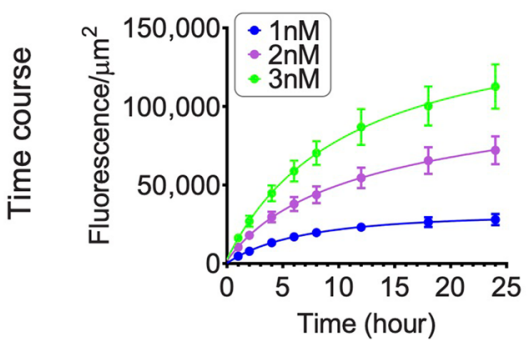

b

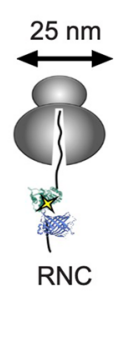

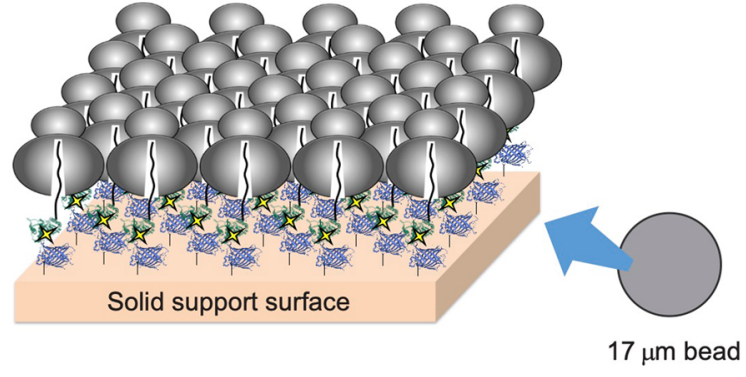

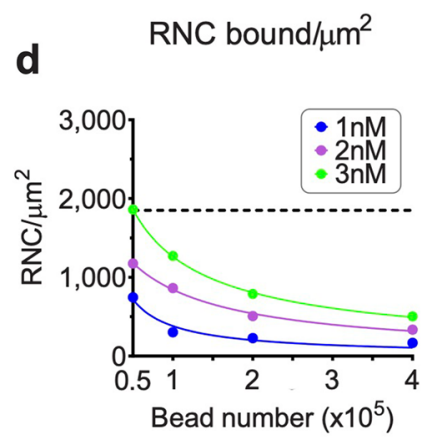

g

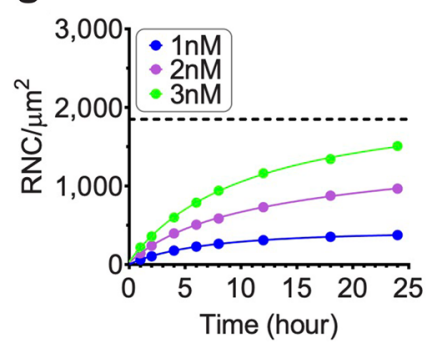

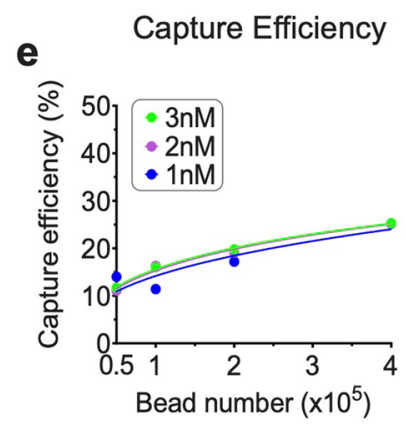

h

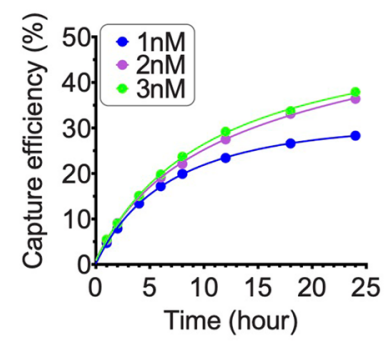

Figure 2. RNC capture is dependent on RNC concentration, binding time, and bead number. (a) Time course of bead binding for $2 \times 10^{5}$ of $17 \mu \mathrm{m}$ beads and $2 \mathrm{nM}$ purified RNCs (His ${ }_{10}$-CFP-NBD1, donor only) \pm RNase A treatment. (b) Cartoon depicting ribosome binding geometry on solid support surface assuming smooth surface. Diameter of ribosome is approximately $25 \mathrm{~nm}$, yielding RNC saturation density of: $\eta_{\mathrm{h}}(1 \mu \mathrm{m})^{2} /(\pi$ $\left.r^{2}\right)=1,850$ ribosomes $/ \mu \mathrm{m}^{2}$, where $\eta_{\mathrm{h}}$ is the coefficient of densest packing of circle in the plane, and $r$ is radius of ribosome. (c-e) Effect of bead number on RNC binding. 1, 2, or $3 \mathrm{nM} \mathrm{RNCs} \mathrm{(His}{ }_{10}$-CFP-NBD1, donor only) were incubated with $0.5-4 \times 10^{5}$ of $17 \mu \mathrm{m}$ beads for $6 \mathrm{~h}$. (f-h) Effect of incubation time on RNC binding. 1, 2, or $3 \mathrm{nM} \mathrm{RNCs}$ ( $\mathrm{His}_{10}$-CFP-NBD1 donor only) incubated with $2 \times 10^{5}$ of $17 \mu \mathrm{m}$ beads for times indicated. Fluorescence intensity of $17 \mu \mathrm{m}$ beads in panels $\mathbf{c}$ and $\mathbf{f}$ are shown in mean $\pm S D(n=\sim 400$ beads). Binding density in panels $\mathbf{d}$ and $\mathbf{g}$ was calculated using number of protein molecules bound per total calculated surface area of beads added. Dotted line in panels $\mathbf{d}$ and $\mathbf{g}$ indicates theoretical RNC saturation density as described in panel $\mathbf{b}$. Capture efficiency in panels $\mathbf{e}$ and $\mathbf{h}$ was calculated by RNCs bound/RNCs in binding reaction.

$$
\mathrm{E}_{\mathrm{FRET}}(\%)=1-\mathrm{F}_{\mathrm{DA}} / \mathrm{FD} \times 100
$$

(where $\mathrm{E}_{\mathrm{FRET}}=\mathrm{FRET}$ efficiency, $\mathrm{F}_{\mathrm{DA}}=$ fluorescence intensities of donor in the presence of acceptor, and $\mathrm{F}_{\mathrm{D}}=$ fluorescence in the presence of donor alone). Note that fluorescence of the acceptor is negligible under these conditions as described previously and therefore was not needed for determining FRET ${ }^{1}$. Solution-based measurements yielded a calculated $\mathrm{E}_{\mathrm{FRET}}$ of $79 \pm 0.5 \%$ (Fig. 3b), which is similar to previous reports and supported by theoretical distance estimates ${ }^{1}$. FRET values were calculated from beads containing immobilized RNCs prepared from matched translation reactions with $\mathrm{D}$ and $\mathrm{D}+\mathrm{A}$ incorporated probes $(\sim 10017 \mu \mathrm{m}$ beads for $\mathrm{D}$ and $\mathrm{D}+\mathrm{A}$ samples) based on mean $F_{D}$ and $F_{D A}$ per unit surface area (fluorescence units $/ \mu \mathrm{m}^{2}$ ). Results of these calculations yielded $\mathrm{E}_{\mathrm{FRET}}$ of $80 \pm 0.2 \%$ in excellent agreement with solution-based measurements (Fig. 3c,d). Both solution and immobilized RNCs yielded FRET values that were somewhat higher than previously reported ${ }^{1}$, likely due to minor variations in experimental conditions and RNC quantitation. The correlation between solution-based measurements and solid-support measurements, indicate that fluorescence measurements and FRET values were relatively unaffected by conditions used for immobilization.

Previously ${ }^{1,2}$, we showed that during synthesis of the NBD1 N-terminal subdomain (CFTR residues 389 to 491), sequestration of C-terminal residues inside the ribosome retains the nascent chain in an unfolded conformation and that folding occurs abruptly as residues 500-550 exit the ribosome tunnel. To investigate whether 
a

$\mathcal{r}\left[{ }^{14} \mathrm{C}\right]$ Lys $\quad \mathcal{Y}$ ENBD-[ $\left.{ }^{14} \mathrm{C}\right]$ Lys

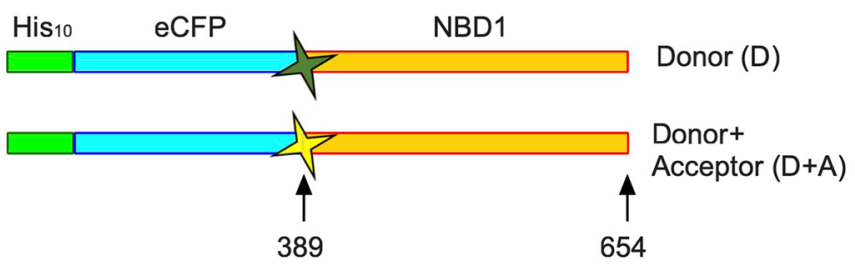

b

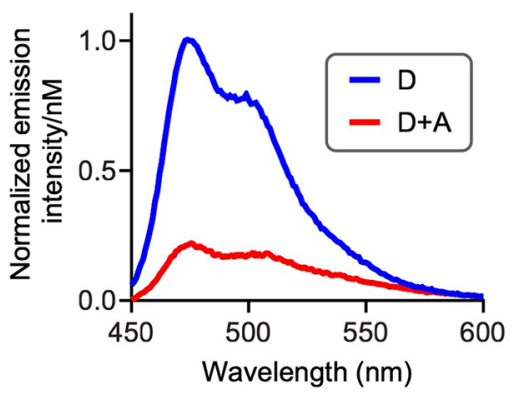

C

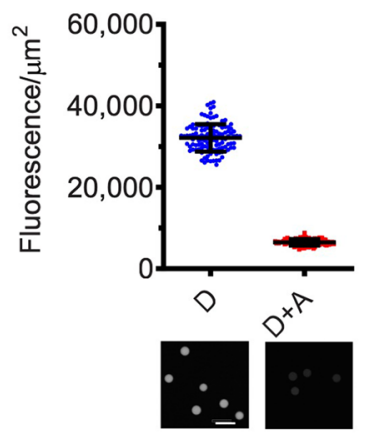

d

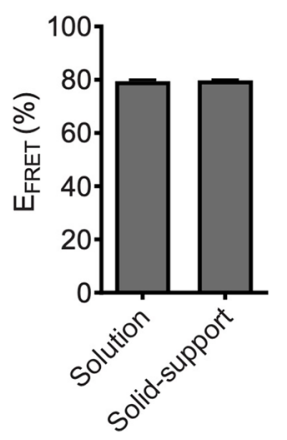

Figure 3. Solution FRET and Solid-support FRET of immobilized RNCs. (a) Schematic of His ${ }_{10-}$ CFP-NBD1 construct showing approximate location of acceptor dye residue (389) and truncation site residue (654). (b) Graph showing CFP fluorescence emission spectra in solution in D and D+A samples. (c) Graph showing fluorescence intensity of $17 \mu$ m beads (mean $\pm S D, n=118$ or 108 for $\mathrm{D}$ or $\mathrm{D}+\mathrm{A}$ beads, respectively). Raw images of beads containing D or D + A RNCs is shown below graph. Scale bar, $50 \mu \mathrm{m}$. (d) Graph showing Solution $\mathrm{E}_{\mathrm{FRET}}$ and Solid-support $\mathrm{E}_{\mathrm{FRET}}$. Data are mean $\pm \mathrm{SEM}, \mathrm{n} \geq 3$ independent experiments. Solution $\mathrm{E}_{\mathrm{FRET}}$ was calculated from D, D + A, and blank samples based on CFP fluorescence intensity at $\lambda \mathrm{em}=475 \mathrm{~nm}(\lambda \mathrm{ex}=430 \mathrm{~nm})$ as described in "Methods" section. Solid-support $\mathrm{E}_{\mathrm{FRET}}$ was calculated from averaged bead fluorescence intensity in panel (c).

RNC immobilization impacts the NBD1 folding status, nascent polypeptides containing an acceptor probe at residue 450 were truncated at residues 500 or 550 , and $\mathrm{E}_{\mathrm{FRET}}$ was determined before and after ribosome release (Supplementary Fig. S4a). Consistent with solution-based results (Supplementary Fig. S4b), immobilized ribosome-bound polypeptides truncated at residue 500 exhibited low $\mathrm{E}_{\mathrm{FRET}}$ values $(12 \pm 4.2 \%)$, and $\mathrm{E}_{\mathrm{FRET}}$ increased 2.5 fold following release from ribosome by RNase digestion (to $31 \pm 3.3 \%$ ) (Supplementary Fig. S4c). Similarly, at truncation 550, the N-terminal subdomain had already folded and little increase in FRET was observed upon ribosome release. Thus, RNC immobilization does not substantially impact NBD1 folding or affect the ability to detect changes in NBD1 conformation in ribosome bound and unbound states. In addition, RNC conformation was remarkably stable following storage at $-80^{\circ} \mathrm{C}$ (Supplementary Fig. S5), thus enabling large batch preparation preformed RNCs for subsequent screening efforts. These results are also consistent with previous work demonstrating that ribosome-bound folding intermediates are remarkably stable ${ }^{44}$ in isolation buffer and provide a practical and scalable method to analyze transient folding intermediates.

Detection of a cotranslational folding defect by solid-support FRET. Numerous mutations within NBD1 cause CF by disrupting NBD1/CFTR folding and/or intracellular trafficking ${ }^{21-23,46}$. Recently, we showed that A455E cotranslationally perturbs NBD1 $\alpha$-helical subdomain folding with the most profound effect observed for nascent chains truncated at residue $654^{24}$. Consistent with solution-based measurements, A455E NBD1 also yielded significantly lower $\mathrm{E}_{\mathrm{FRET}}(29 \pm 0.1 \%)$ compared to wild-type $(36 \pm 0.5 \%)$ (mean $\left.\pm \mathrm{SEM}\right)$ in the solid-support FRET system (Fig. 4). Thus, immobilized RNCs reproduce mutation-induced deviations in the cotranslational folding pathway. In addition, we previously demonstrated that a combination of suppressor mutations (S492P and I539T (PT)) genetically corrected the cotranslational folding defect induced by A455E and partially restored folding of full-length CFTR trafficking in cells ${ }^{24}$. A suppressor mutation S492P is predicted to increase rigidity between the $\mathrm{N}$-terminal and the $\alpha$-helical subdomains ${ }^{47}$, and a well-studied suppressor mutation $\mathrm{I}_{539 \mathrm{~T}^{48}}$ is known to increase thermal stability of F508del NBD1. Like the PT, numerous suppressor mutations have been studied to understand CFTR/NBD1 folding defects, mechanism of action of corrector molecules, and used in some HTS systems ${ }^{22,23,42,49}$. As shown in Fig. 4c, the PT suppressor mutations also corrected the folding defect of A455E NBD1 observed by solid-support FRET ${ }^{24}$. These results suggested that immobilized 
a

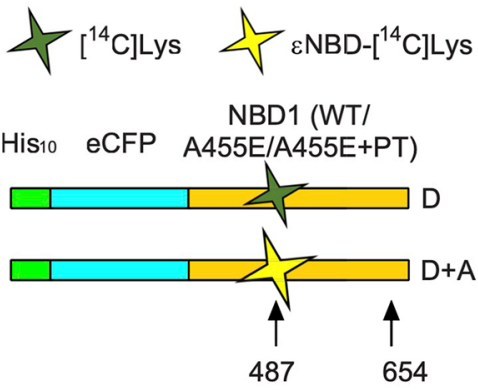

b

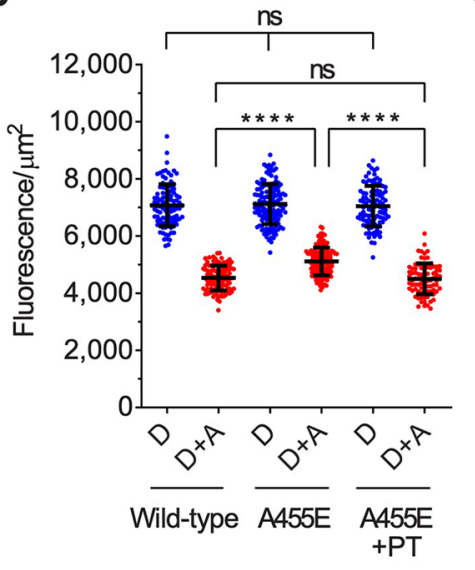

C

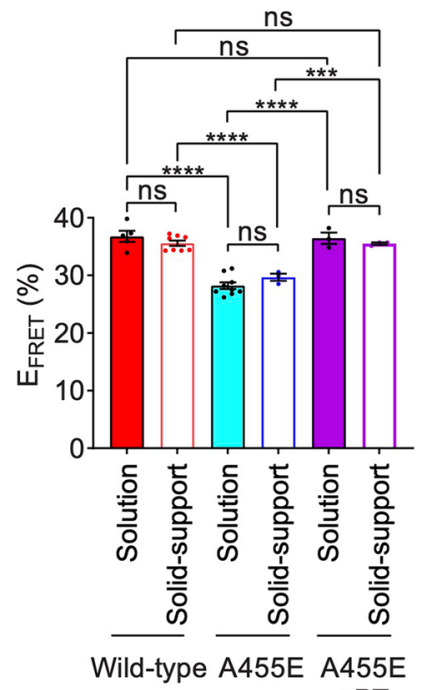

Figure 4. FRET analysis of wild-type or A455E NBD1 folding. (a) Schematic of $\mathrm{His}_{10}$ CFP-NBD1 (wild-type/ A455E/A455E + PT) constructs showing approximate location of acceptor dye and truncation residues 487 and 654, respectively. (b) Graph showing fluorescence intensity of individual D or D+A beads for wild-type, A455E, or A455E + PT constructs (acceptor at 487 and truncation at 654) obtained from individual experiments in 384-well format (mean $\pm \mathrm{SD}, \mathrm{n}=81$-142 beads). (c) Graph showing $\mathrm{E}_{\mathrm{FRET}}$ values obtained from individual experiments of solution or solid-support FRET assay in 384-well format for wild-type, A455E, or A455E + PT constructs indicated (mean \pm SEM, $\mathrm{n}=3-9$ individual experiments). Two-tailed unpaired student's t-test, ${ }^{* * *} \mathrm{p}<0.001,{ }^{* * * *} \mathrm{p}<0.0001$, n.s. $>0.05$.

A455E NBD1 cotranslational folding intermediates could be used to screen for small molecules that might interact with the nascent polypeptide and restore folding similar to the PT suppressor mutations.

Note that during our study, GE introduced a software update algorithm for flat field correction in the InCell system that reduced the values of reported fluorescence signal. However, this software change had no impact on FRET values (see Supplementary Fig. S6 and methods for details).

Optimization of FRET detection in multi-well plates. Immobilized RNCs were initially tested in 96 -well and 384-well plates by dispensing 1,000 beads in $200 \mu \mathrm{l} /$ well or 500 beads in $50 \mu \mathrm{l} /$ well, respectively, containing $\mathrm{His}_{10}$-CFP-NBD1 RNCs (acceptor probe at residue 450 and truncation at residue 550) into 48 wells (24 wells each for D and DA beads). Note that wild-type constructs were used for 96-well and 384-well plate assays as these trials were performed prior to the study of A455E cotranslational folding defect ${ }^{24}$. Four images were taken of each well, and approximately 30 beads were selected per well (after eliminating unfocused beads and artifacts) to quantify mean fluorescence intensity (see "Methods" section for details). Each matched pair of wells (D and D +A) therefore yielded a single FRET measurement (Supplementary Fig. S7). While 96-well and 384-well plate formats yielded nearly the same $\mathrm{E}_{\mathrm{FRET}}$ values $(50.6 \pm 2.0 \%$, and $50.3 \% \pm 1.4 \%$, respectively), the 384 -well format showed less variation. Bead titration revealed that variation in fluorescence signal and $\mathrm{E}_{\mathrm{FRET}}$ values improved as the number of images ( 9 per well) and beads ( $>400 /$ well) were increased in the 384-well plate format (Supplementary Fig. S8). Among known ABC transporters, NBD1 contains a unique unstructured regulatory region (RI: residues 405-436) of uncertain function. When deleted $(\Delta \mathrm{RI})$, maturation, stability, and function of wildtype CFTR are improved and F508del CFTR function and trafficking in cells are partially restored ${ }^{50,51}$. RI deletion also improves solubility and thermostability of the isolated NBD1 domain ${ }^{20,51,52}$ through the stabilization of the $\alpha$-subdomain and $\alpha / \beta$-core. We therefore compared the efficiency of wild-type and $\triangle \mathrm{RI}$ NBD1 folding in 384 well format. $\mathrm{E}_{\mathrm{FRET}}$ values were $51.7 \pm 0.7 \%$ for wild-type, and $61.6 \% \pm 0.6 \%$ for $\Delta \mathrm{RI}$ (Supplementary Fig. S9). Comparison of $\mathrm{E}_{\mathrm{FRET}}$ values yielded 0.70 of estimated $\mathrm{Z}^{\prime}$ factor ${ }^{53}$, consistent with robust assay reproducibility. Importantly, $\geq 8 \%$ FRET difference between negative and positive controls was needed to obtain $Z^{\prime}$ factor $>0.5$ based on this result. For practical purposes, wild-type and $\Delta$ RI RNCs were used as negative and positive controls for the Z' factor estimation.

To improve scalability, we also evaluated a 1,536-well plate format: $2 \mathrm{nM}$ RNCs containing A455E NBD1 (acceptor probe at residue 487, and truncation at residue 654) were incubated with $1 \times 10^{6} 17 \mu \mathrm{m}$ beads in 800 $\mu \mathrm{L}$ binding buffer for over-night $(16 \mathrm{~h})$. Reproducibility improved as the number of bead/well was increased to 600 (Fig. 5). Moreover, the average of two adjacent FRET measurements improved reproducibility of $\mathrm{E}_{\mathrm{FRET}}$ value relative to individual FRET measurements (Fig. 5c). Given the small well area $\mathrm{E}_{\mathrm{FRET}}$ values required only 1-image/well, substantially reducing the length of imaging time to $38 \mathrm{~min}$ for 768 FRET measurements versus 77 min for 192 FRET measurements in 384-well format (9-images/well). To account for imaging multiple plates over several hours, we showed that prolonged incubation at room temperature did not affect RNC stability in 


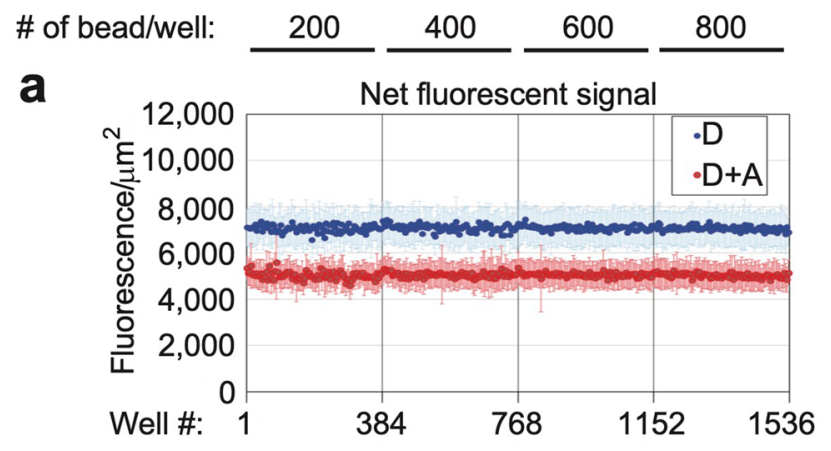

b

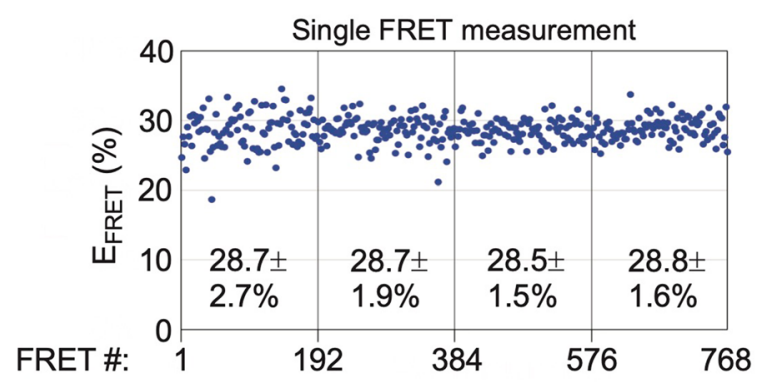

C

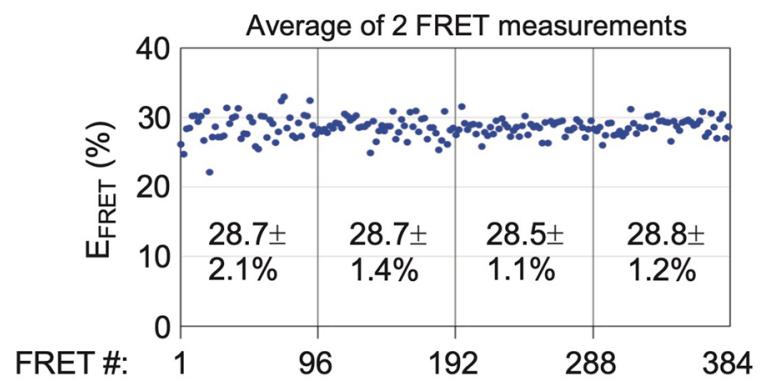

Figure 5. Bead titration in 1,536-well format. (a) Individual dots show mean fluorescence intensity \pm SD for $\mathrm{D}$ and $\mathrm{D}+\mathrm{A}$ samples bound to $17 \mu \mathrm{m}$ beads in matched paired wells of a 1,536-well plate. Number of beads in each well is indicated at top. (1-image/well $=19 \%$ of total well area). (b \& c) Individual $\mathrm{E}_{\mathrm{FRET}}$ values calculated from single $D$ and $D+A$ pair of wells $(\mathbf{b})$, or average of two $D$ and $D+A$ pairs $(\mathbf{c})$. Mean $E_{\mathrm{FRET}} \pm S D$ values were calculated for each bead binding condition $(n=192$ single $D$ and $D+A$ pair or 96 average of two $D$ and $D+A$ pairs in panels $\mathbf{b}$ or $\mathbf{c}$, respectively).

dilution buffer (Supplementary Fig. S10). Thus, multiple plates could be prepared and stored at room temperature for up to $24 \mathrm{~h}$ until imaging with little impact on data quality.

FRET-based high throughput screening. We tested a diverse 50,000 small-molecule library from ChemDiv, Inc. (https://www.chemdiv.com/) as a pilot HTS in $0.1 \%$ DMSO condition using batched and frozen RNCs expressing A455E NBD1 (acceptor probe and truncation sites at residues 487 and 654, respectively) in 1,536well format. DMSO is well tolerated at the HTS condition (0.1\% DMSO) (Supplementary Fig. S11). Compounds were tested in duplicate using paired $\mathrm{D}$ and $\mathrm{D}+\mathrm{A}$ wells, thus allowing 320 compounds to be tested per plate (Supplementary Fig. S12). Figure 6 shows a representative plate analysis. To compensate for small molecule artifacts, compounds that increased or decreased baseline CFP fluorescence intensity and/or increased background signal intensity, were eliminated based on the following criteria as quality control (QC) tests: $\geq \pm 10 \%$ change in fluorescence intensity of $\mathrm{D}$ beads or $\geq 20 \%$ change in background signal intensity (Fig. $6 \mathrm{a}-\mathrm{c}$ ). These criteria were chosen based on variations of bead signals and background signals. This resulted in approximately $10 \%$ of data being unusable. Note that no small molecules have been reported to directly interact with NBD1 cotranslational folding intermediates, therefore, this pilot HTS was performed without positive controls. Instead, DMSO baseline control data was used to identify hit compounds on the basis of averaged $(\mathrm{N}=2) \mathrm{E}_{\mathrm{FRET}}$ measurements that showed $> \pm 3$ SD relative to DMSO control (Fig. 6d). Figure 7a shows raw $\mathrm{E}_{\mathrm{FRET}}$ values for $\sim 50,000$ compounds and 133 primary hit compounds ( $0.26 \%$ hit ratio) obtained from 157 1,536-well plates. 68 compounds out of the 133 hit compounds showed increase in $\mathrm{E}_{\mathrm{FRET}}$ whereas other 65 compounds decreased $\mathrm{E}_{\mathrm{FRET}}$. $\mathrm{E}_{\mathrm{FRET}}$ values were remarkably consistent across plates measured on different days (Fig. $7 \mathrm{~b}$ ) as revealed for robust assay reproducibility (coefficient of variation $(C V)=3.9 \pm 0.7 \%$ and signal to background ratio $(S / B)=2.62 \pm 0.12$ ) (Fig. $7 \mathrm{c}, d)$. 
a

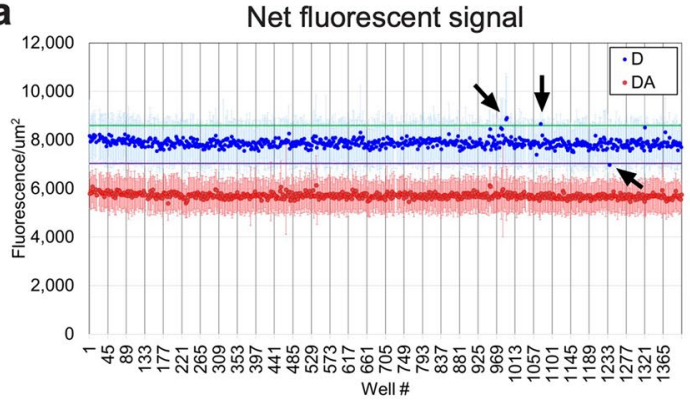

C

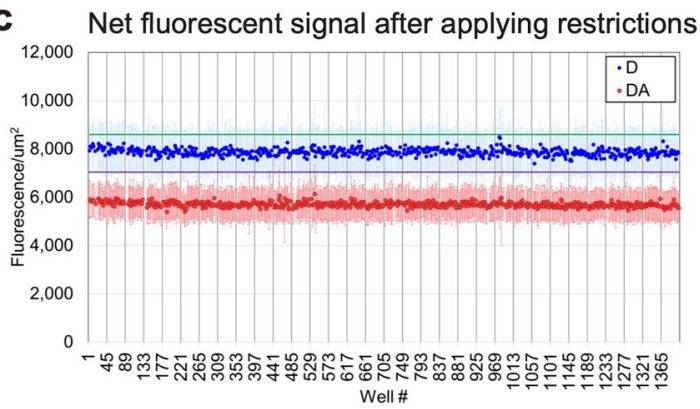

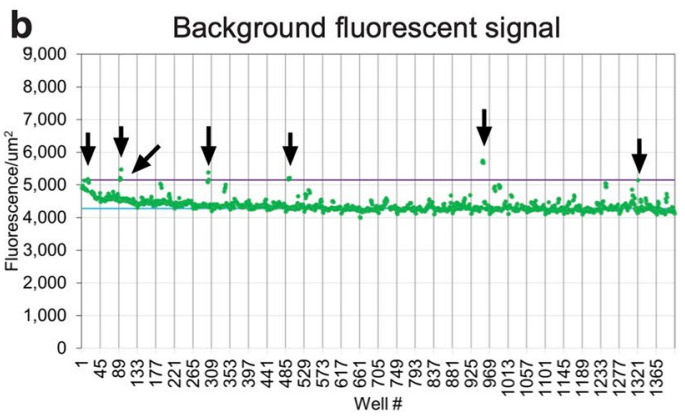

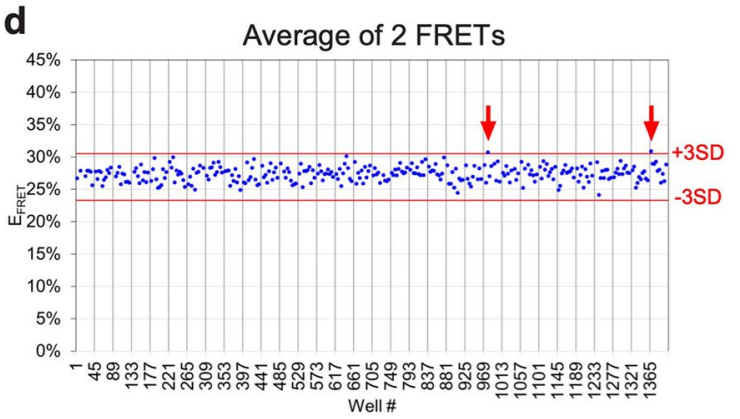

Figure 6. Representative plate analysis for 1,536-well HTS. (a) Graph showing bead fluorescence intensity of individual $\mathrm{D}$ (blue) or $\mathrm{D}+\mathrm{A}$ (red) wells (mean $\pm \mathrm{SD}, \mathrm{n}=\sim 64$ ) in 1,536-well format. Arrows indicate sample where $\mathrm{D}$ fluorescence is above 1.1-fold (green line) or below 0.9-fold (magenta line) of mean of DMSO baseline control. (b) Graph showing background fluorescence intensity of each well. Black arrows indicate wells in which background signal was above 1.2-fold (magenta line) of mean background signal of DMSO baseline control (cyan line). (c) Graph showing fluorescence intensity of individual D (blue) or D + A (red) wells (mean \pm SD, $\mathrm{n}=\sim 64$ ) after applying following two restrictions; donor fluorescence intensity was between 1.1-fold or 0.9-fold of DMSO control (shown in panel a); and background signal was below 1.2 fold of DMSO control (shown in panel b). (d) Individual blue dots represent $E_{F R E T}$ values calculated based on average of two $D$ and $D+A$ pairs from panel $\mathbf{c}$ for each compound. Red arrows indicate a hit compound showing averaged FRET values outside of \pm 3 standard deviation (SD) of DMSO control (red lines in panels $\mathbf{d}$ ).

All 133 primary hit compounds were subsequently tested in 6-point dose-response studies and two compounds were found to reproducibly altered $\mathrm{E}_{\mathrm{FRET}}$ values (Fig. 8a-d). Compound 0237292 resulted in a dose dependent increase in $\mathrm{E}_{\mathrm{FRET}}\left(5 \%\right.$ at $\geq 4 \mu \mathrm{M}$ with an $\mathrm{EC}_{50}$ of $\left.1.7 \mu \mathrm{M}\right)$ (Fig. 8c). In contrast, compound 0256757 decreased $\mathrm{E}_{\mathrm{FRET}}$ values by $4 \%$ at $40 \mu \mathrm{M}$ (Fig. 8d). To distinguish whether the observed changes in $\mathrm{E}_{\mathrm{FRET}}$ reflected a direct effect on NBD1 folding, we used a previously described control NBD1 construct containing the acceptor probe at residue 389 (CFP fusion site). Recall that $\mathrm{E}_{\mathrm{FRET}}$ for this construct is dependent only on the tether length. Therefore, compounds that specifically impact NBD1 folding should have no effect on $\mathrm{E}_{\mathrm{FRET}}$. As shown in Fig. 8e, compound 0237292 did not substantially change $\mathrm{E}_{\mathrm{FRET}}$ for the control construct, whereas compound 0256757 decreased $\mathrm{E}_{\mathrm{FRET}}$ values to the same extent as were observed when the acceptor probe located at residue 487 (Fig. 8f). Thus, we suspect that compound 0256757 exerts its effect on fluorescent properties the donor and/or acceptor probes rather than NBD1 folding per se. Because the only difference between the control construct and screening constructs is the location of the probe with in the NBD1 domain (residues 389, and 487, respectively), we also conclude that compound 0237292 likely interacts with the nascent NBD1 polypeptide to produce the resulting increase in FRET. A455E mutation disrupts $\alpha / \beta$-core formation during NBD1 cotranslational folding as described in our previous study using RNCs containing an acceptor probe at residue $567^{24}$ (Supplementary Fig. S13). To confirm whether compound 0237292 corrects folding disruption of $\alpha / \beta$-core, we next tested compound 0237292 using the A455E NBD1 RNC construct containing an acceptor probe at residues 567 (truncation sites 674) as a secondary confirmation assay. However, compound 0237292 did not restore $\mathrm{E}_{\mathrm{FRET}}$ (Fig. 8g). Taken together, compound 0237292 partially corrected A455E NBD1 folding intermediates, suggesting that additional restorations are needed to fully correct A455E folding defects.

Aside from the compound 0237292 identified in this pilot HTS, no small molecules have been shown to directly interact with NBD1 cotranslational folding intermediates. However, certain compounds, including several approved drug components, have been proposed to either interact with full-length CFTR or to influence cotranslational folding outcome through unknown mechanisms. We therefore examined 5-bromoindole-3-acetic acid (BIA), a reported NBD1 stabilizer ${ }^{54}$, and other corrector molecules including VX-809, VX-661, and VX-445 in our cotranslational folding assay. Supplementary Figure S14 showed that none of these compounds restored $\mathrm{E}_{\mathrm{FRET}}$ for RNCs of A455E NBD1, although BIA decreased CFP fluorescence intensity at the higher concentrations $(\geq 4 \mathrm{mM})$.

We next tested whether compound 0237292 would correct folding of A455E NBD1 or full-length CFTR. As shown in Fig. 8h, compound 0237292 did not influence either wild-type or A455E CFTR trafficking in HEK293 
a

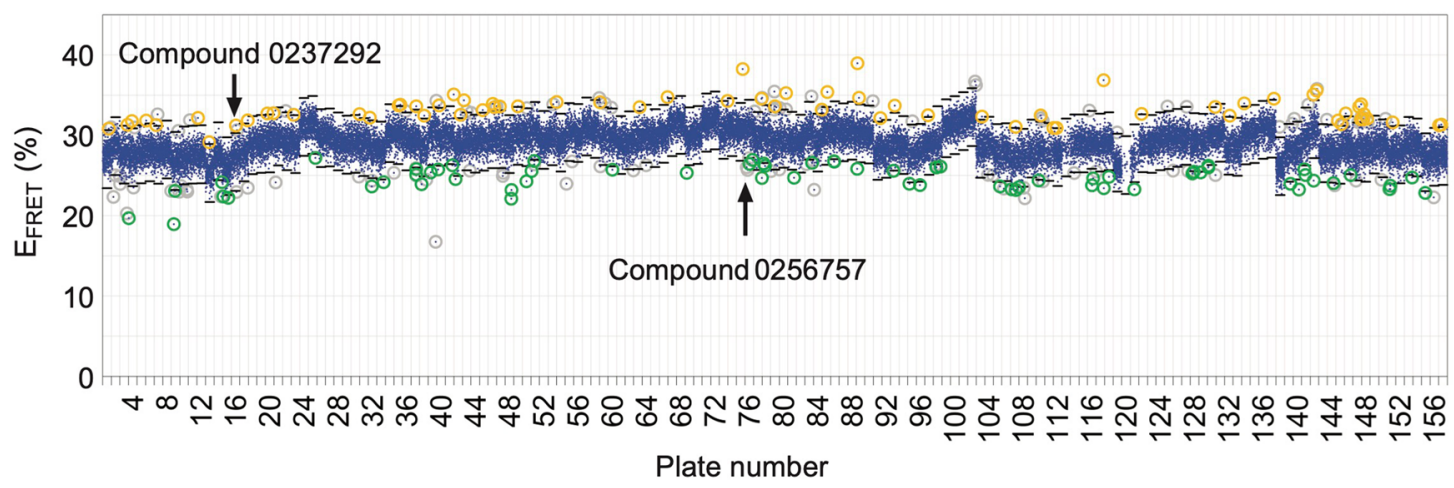

b

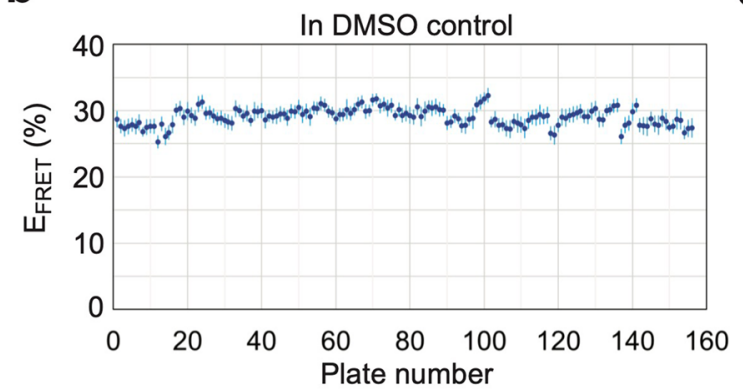

C

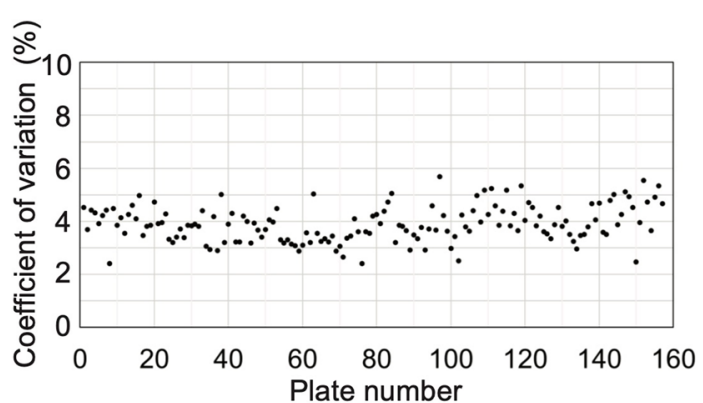

d

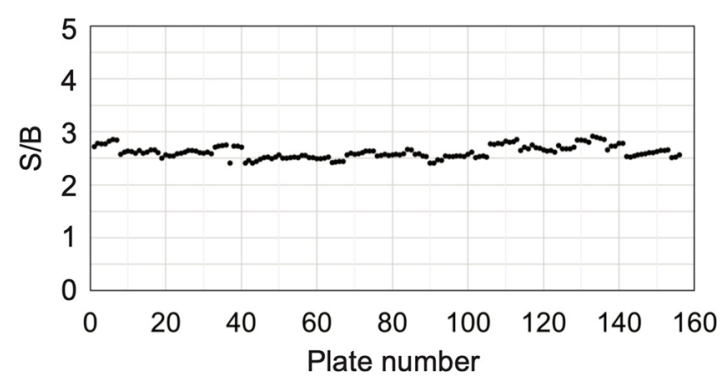

Figure 7. HTS results. (a) Raw FRET results for A455E NBD1 (acceptor and truncation at residues 487 and 654) screen using a 50,240 small molecule diversified library. Blue dots represent each averaged $\mathrm{E}_{\mathrm{FRET}}$ value calculated based on two D and D + A pairs, and measured for compounds from 157 plates (1,536-well plate format, 600 beads/well, 320 compounds/plate). Black lines show $\pm 3 S D$ for each plate. 133 primary hit compounds are indicated in orange circles (increase in FRET) or green circles (decrease in FRET) $(0.26 \%$ hit ratio). Gray circles indicate averaged FRET values outside of \pm 3 SD but either or both single FRET values inside of $\pm 1.75 \mathrm{SD}$. (b) Graph showing $\mathrm{E}_{\mathrm{FRET}}$ values (mean $\pm \mathrm{SD}, \mathrm{n}=32$ ) of DMSO control for each plate. (c-e) Black dots represent coefficient of variation ( $\mathrm{SD} /$ mean) of $\mathrm{E}_{\mathrm{FRET}}$ values (c), or signal to background (mean $\mathrm{D}$ signal/ mean background) (d) for each plate.

cells as indicated by lack of Golgi-processed, Band C protein. VX-809 (lumacaftor), which partially restores CFTR folding, was added if compound 0237292 showed any synergistic effects with VX-809. However, addition of $3 \mu \mathrm{M}$ VX-809 to compound 0237292 treatment failed to show any additional effects. Pulse-chase experiments also revealed that 0237292 had no significant effect on stability of wild-type or A455E for the isolated NBD1 domain (two-tailed student's t-test for protein half-life, $\mathrm{p}=0.32$ or 0.64 , respectively) (Fig. $8 \mathrm{i}, \mathrm{j}$ ).

\section{Discussion}

In this study we report a highly sensitive solid-support assay system well suited for high throughput screening that is capable of detecting subtle conformational changes in ribosome bound nascent proteins. Quantitative fluorescence imaging of immobilized RNCs generated by cell free in vitro translation allowed detection of approximately 0.4 attomole RNCs/bead with high degree of reproducibility in 1536 well format $(\mathrm{CV}=3.9 \pm 0.7 \%)$. RNCs isolated from cell free in vitro translation reactions are stable at room temperature and amenable to cryopreservation, enabling scaling efforts needed for screening consistency. A pilot HTS of approximately 50,000 small molecules in 1,536-well plate format demonstrated suitability for screening small molecules that interact with, and alter nascent protein structure. These results provide a unique HTS method to capture folding intermediates 
a

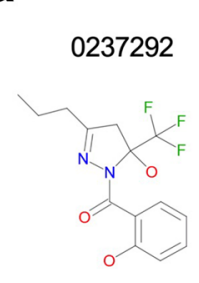

b

0256757

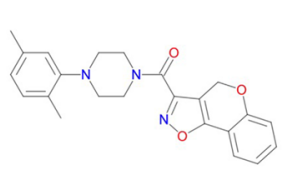

C

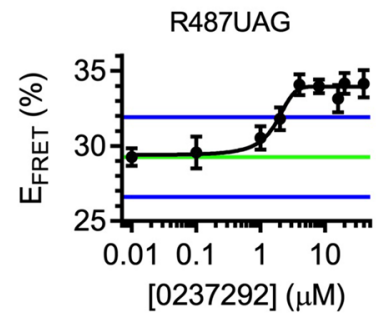

d

R487UAG

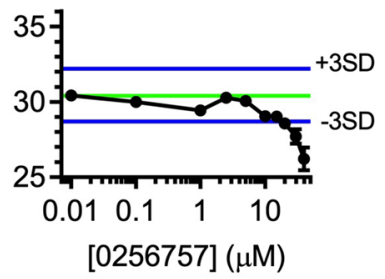

e

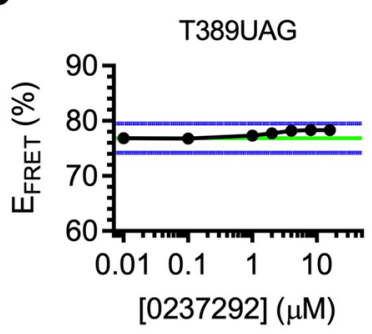

f

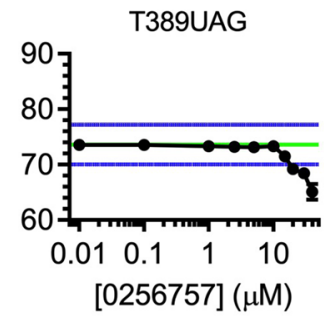

g

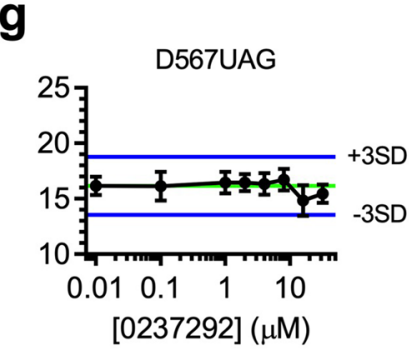

h
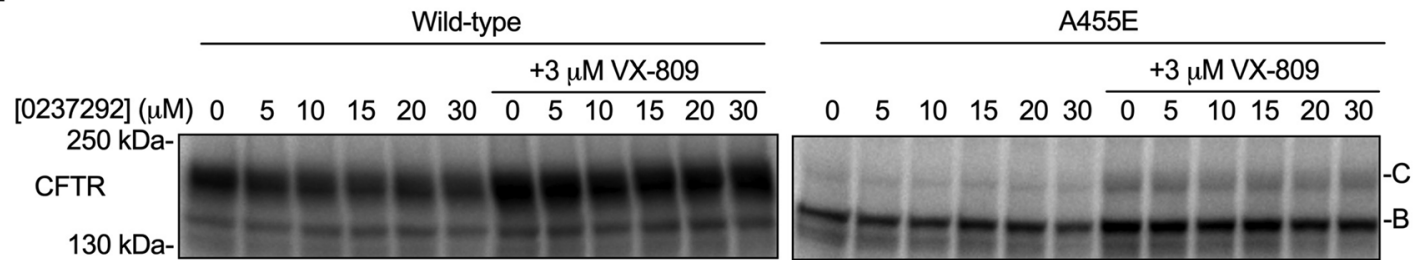

$\beta$-actin

$36 \mathrm{kDa}$

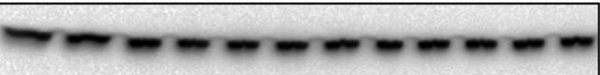

\section{要}

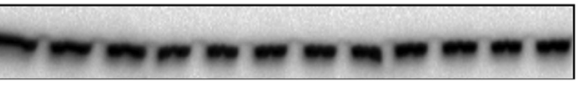

i

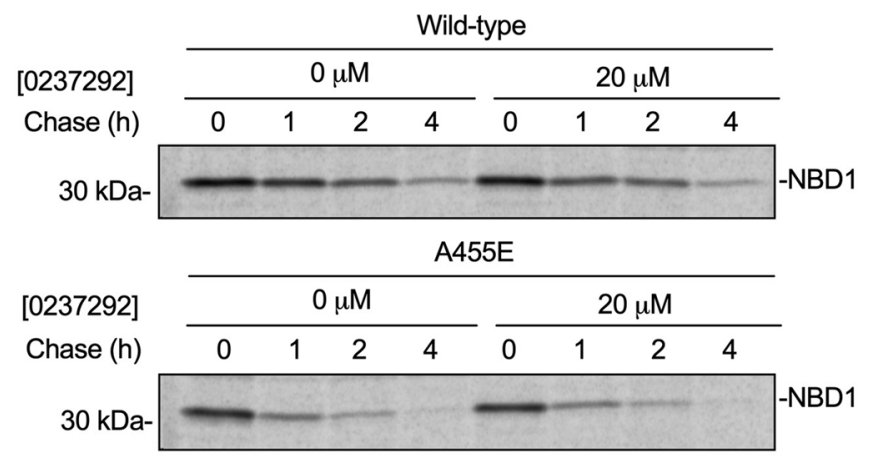

Figure 8. Hit confirmation. (a, b) Chemical structures of compound 023792 (a) or compound 0256757 (b). (cg) Dose response results for compound $0237292(\mathbf{c}, \mathbf{e}, \mathbf{g})$ or compound 0256757 (d,f) measured by solid-support FRET using A455E NBD1 RNCs with acceptor dye inserted at residue 487 (c,d), 389 (e,f), or 567 (g). Data are mean \pm SEM, $n \geq 3$ independent experiments. (h) Immunoblot of CFTR wild-type or A455E with $0-30 \mu \mathrm{M}$ compound 0237292 with or without $3 \mu \mathrm{M}$ VX-809 treatment expressed in human embryonic kidney (HEK) 293 cells showing no effect of compound 0237292 on both wild-type or A455E CFTR trafficking. Core glycosylated (band B) and mature CFTR (band C) are indicated. Uncropped blots in Supplementary Figure S15, S16. (i,j) Effect of compound 0,237,292 $(20 \mu \mathrm{M})$ on stability of wild-type or A455E NBD1 in HEK 293 cells, determined by $\left[{ }^{35} \mathrm{~S}\right]$ Met-labeled NBD1 immunoprecipitation. Uncropped gels in Supplementary Figure S17. Graph shows percentage of $\mathrm{NBD} 1$ recovered relative to $\mathrm{T}=0$ (mean $\pm \mathrm{SEM}, \mathrm{n}=3$ independent experiments).

of both wild-type and genetic mutants that are normally present only transiently during synthesis. In addition, it is also suited for full length or truncated proteins regardless of their ribosome attached or released state. 
Our pilot HTS identified a single validated hit $(\# 0,237,292)$ that restored $E_{\text {FRET }}$ for RNCs of A455E NBD1 containing an acceptor probe at residue 487 to the same extent as previously described suppressor mutations (Fig. 8c, e). Unfortunately, activity of 0,237,292 did not translate into improved CFTR trafficking or NBD1 stability (Fig. $8 \mathrm{~h}-\mathrm{j}$ ). This is perhaps not surprising because compound 0,237,292 partially corrected A455E NBD1 folding defects (Fig. 8g) and the molecule itself could affect numerous other aspects of CFTR folding as has been proposed for compounds that increase NBD1 thermal stability ${ }^{32,54,55}$. Although disappointing, the likelihood of identifying a compound that would act with this specific mechanism of action from a relatively small 50,000 compound library is quite low. Libraries covering a much larger chemical space would likely be needed to identify small molecules that act via such a mechanism.

We acknowledge that one of the most technically challenging aspects of this system involves generation of biochemically uniform cohorts of RNCs with defined fluorophore stoichiometry. Fluorophore incorporation is also restricted to peptide regions that will tolerate modification by a donor and acceptor probe. However, the growing number of studies using FRET to monitor conformational changes associated with folding, function, enzyme activation, posttranslational modification, and oligomerization, support a broader applicability for this approach $^{56-61}$.

It is also important to note that techniques reported here could potentially be further optimized to improve in vitro translation yield, solid support capture efficiency, and uniformity in substrate binding. Of the 12 binding strata tested, we found that Ni-IDA $17 \mu \mathrm{m}$ beads gave the best signal, binding efficiency, and fluorescence reproducibility. Bead-binding conditions shown here for $17 \mu \mathrm{m}$ beads yielded quite acceptable signal-to-background ratios and achieved surface densities of $250-500 \mathrm{RNCs} / \mu \mathrm{m}^{2}$. This represents $13-27 \%$ theoretical geometric saturation based on ribosome cross-sectional area and an effective increase in RNC concentration approximately 100fold greater than solution studies ( $\sim 2 \mathrm{nM}$ in solution vs. $0.16-0.31 \mu \mathrm{M}$ bound). RNC immobilization therefore enabled us to achieve sub-attomolar detection sensitivity. Typical translation reactions of the His $_{10}$-CFP-NBD1 $^{-N}$ A455E (acceptor 487 and truncation 654) construct used in this study (6 ml cell free in vitro translation reactions) yield sufficient material (42 picomol of purified RNCs) to generate approximately 26 million beads ( 13 million each of $\mathrm{D}$ and $\mathrm{D}+\mathrm{A}$ beads), which when plated at 600 beads/well in 1,536-well plate, provides sufficient material for 21,500 single FRET measurements. Although this method relies on image-based readout like high-content screening (HCS) systems, with an image time of $0.5 \mathrm{~s}$ per well (1-image/well) in 1,536-well plate format using the GE IN Cell Analyzer 2200, approximately 1 million small molecule compounds could theoretically be screened in 3 months ( 36 assay plates a day), which meets the HTS standard ( $>100,000$ compounds screen $)^{62}$. We would also note that in our experience, translation yields vary for different protein constructs, and are dependent upon the length of transcript and location and readthrough efficiency of the stop codon ( $30 \%$ in this study). However, our results reflect reasonable estimates that were readily obtained and which could be further optimized or scaled if needed for industrial screening programs.

Important practical considerations include: (i) the time required from RNA transcription to protein synthesis and RNC purification is approximately $4 \mathrm{~h}$; (ii) no large-scale protein purification is required; (iii) partial length and even misfolded proteins are stably retained, while bound to the ribosome, in a non-aggregated, foldingcompetent state for prolonged periods of time ${ }^{1,2,44}$. Immobilized RNCs are therefore well suited for substrates that cannot be efficiently expressed in cells and/or are not amenable to traditional biochemical analyses for other reasons. Importantly, there are currently no existing HTS assays to evaluate cotranslational folding, which represents a potential novel approach for developing new treatment strategies ${ }^{24}$. Thus, the HTS assay reported here could be useful in studying additional CFTR mutations or adapting for use in other protein folding disorders or drug discovery efforts.

\section{Methods}

Plasmids. eCFP-NBD1 fusion constructs containing UAG stop codons at CFTR residues Thr389, Arg450, or Arg487 were generated in the pSP64 plasmid vector (Promega) as described previously ${ }^{1}$. A His ${ }_{10} \operatorname{tag}\left(5^{\prime}\right.$-catcaccatcaccatcaccatcaccatcac) followed by flexible 21 amino acid residues linker (GGS) ${ }_{7}$ was fused the N-terminus of CFP by PCR overlap extension as described elsewhere ${ }^{1}$. A455E mutation was generated in a similar technique ${ }^{24}$. All cloned PCR fragments were verified by DNA sequencing.

In vitro transcription and translation. Noncapped RNA transcripts were synthesized from PCR-amplified DNA templates $(15 \mathrm{ng} / \mu \mathrm{l})$ in $80 \mathrm{mM}$ HEPES-NaOH (pH 7.5); $16 \mathrm{mM} \mathrm{MgCl}_{2} ; 2 \mathrm{mM}$ spermidine; $3 \mathrm{mM}$ each ATP, CTP, UTP, and GTP; $10 \mathrm{mM}$ DTT; $0.2 \mathrm{U} / \mu \mathrm{l}$ RNase inhibitor; and $5 \mu \mathrm{g} / \mathrm{ml}$ SP6 RNA polymerase at $40{ }^{\circ} \mathrm{C}$ for $2 \mathrm{~h}$ as described elsewhere ${ }^{63}$. RNA was precipitated with $3 \mathrm{M} \mathrm{LiCl}$ and $20 \mathrm{mM}$ EDTA at $-20^{\circ} \mathrm{C}$ for $1 \mathrm{~h}$ and centrifuged at $16,000 \times g$ at $4{ }^{\circ} \mathrm{C}$. The RNA pellet was washed three times with $70 \%(\mathrm{v} / \mathrm{v})$ ethanol and then resuspended in RNase free $\mathrm{H}_{2} \mathrm{O}$ and stored at $-80^{\circ} \mathrm{C}$.

Four parallel in vitro translation reactions were performed as described previously ${ }^{1,2}$. Briefly translation was carried out for $72 \mathrm{~min}$ at $24^{\circ} \mathrm{C}$ in reactions containing $60 \mathrm{ng} / \mu \mathrm{l}$ purified RNA, $40 \%$ rabbit reticulocyte lysate (RRL) prepared as described previously ${ }^{63}, 20 \mathrm{mM}$ HEPES-KOH (pH 7.6), $\left.100 \mathrm{mM} \mathrm{KOAc,} \mathrm{1.6-2.0} \mathrm{mM} \mathrm{Mg(OAc)}\right)_{2}$, $50 \mu \mathrm{M}$ each of 20 amino acids, $1 \mathrm{mM}$ ATP, $1 \mathrm{mM}$ GTP, $15 \mathrm{mM}$ creatine phosphate, $2 \mathrm{mM}$ DTT, $0.15 \mathrm{mM}$ spermidine, $40 \mathrm{ng} / \mu \mathrm{l}$ bovine tRNA, $40 \mathrm{ng} / \mu \mathrm{l}$ creatine kinase, $0.12 \mathrm{U} / \mu \mathrm{l}$ RNase inhibitor, and either $1 \mu \mathrm{M}\left[{ }^{14} \mathrm{C}\right]$ Lys-tRNA ${ }^{\text {amb }}$ or $\varepsilon N-7$-nitrobenz-2-oxa-1,3-diazol ( $\left.\varepsilon N B D\right)-\left[{ }^{14} C\right]$ Lys-tRNA ${ }^{\text {amb }}$ prepared as described previously ${ }^{1}$. Matched translation samples were prepared as following: CFP donor only (D)-translated in the presence of $\left[{ }^{14} \mathrm{C}\right]$ Lys-tRNA ${ }^{\mathrm{amb}}$, Donor + Acceptor $(\mathrm{D}+\mathrm{A})$ - translated in the presence of $\varepsilon N B D-\left[{ }^{14} \mathrm{C}\right]$ Lys-tRNA ${ }^{\text {amb }}$, and blank-D and blank-D + A (BD and BDA)-two "blank" (control) reactions in the presence of either $\left[{ }^{14} \mathrm{C}\right]$ Lys-tRNA ${ }^{\text {amb }}$ or $\varepsilon N B D-\left[{ }^{14} \mathrm{C}\right]$ Lys-tRNA ${ }^{\mathrm{amb}}$ were prepared using non-fluorescence eCFP expressing transcripts lacking a UAG codon as described previously ${ }^{2}$. Blank samples were used to quantify the concentration of ribosome nascent 
chain complexes (RNCs) to use for bead binding reactions, and to obtain ${ }^{14} \mathrm{C}$-corrected fluorescence intensity in solution-based FRET measurement. An RNA aptamer that inhibits translation termination factors (eRF1/ eRF3) was added $(0.2-2 \mu \mathrm{M})$ to translation reaction to improve read-through and achieve similar readthrough efficiencies for $\mathrm{D}$ and $\mathrm{D}+\mathrm{A}$ translation reactions ${ }^{2,64}$.

RNCs preparation. RNCs were isolated from translation reactions at $4{ }^{\circ} \mathrm{C}$ by size exclusion column chromatography (Sepharose CL-6B) equilibrated in buffer containing $40 \mathrm{mM}$ HEPES-KOH (pH 7.6), $100 \mathrm{mM}$ $\mathrm{KOAc}$, and $10 \mathrm{mM} \mathrm{MgCl}_{2}$ (column buffer), flash frozen in liquid nitrogen, and store at $-80^{\circ} \mathrm{C}$. RNC concentration was determined by ${ }^{14} \mathrm{C}$ scintillation counting using the following equation:

$$
[\mathrm{RNC}]=\left(\mathrm{cpm}_{\mathrm{S}}-\mathrm{cpm}_{\mathrm{B}}\right) /(\mathrm{CE} \times \mathrm{SA} \times \mathrm{vol})
$$

where [RNC] is the concentration of RNCs (in $\mathrm{nM}$ ), $\mathrm{cpm}_{\mathrm{S}}$ and $\mathrm{cpm}_{\mathrm{B}}$ are ${ }^{14} \mathrm{C}$ counts/min of sample and blank sample ( $D$ and $B D$, or $D+A$ and $B D A$ pair), respectively. $C E$ is counting efficiency (estimated at $95 \%$ for ${ }^{14} \mathrm{C}$ ), $\mathrm{SA}$ is the specific activity of $\left[{ }^{14} \mathrm{C}\right]$ Lys in $\mathrm{dpm} / \mathrm{pmol}$, and vol is the volume of sample in $\mathrm{ml}$.

Solution based fluorescence measurements. Fluorescence measurement and calculation of FRET efficiency were performed as described previously ${ }^{1,2}$. CFP fluorescence emission spectra $(\lambda \mathrm{ex}=430 \mathrm{~nm}$, $\lambda \mathrm{em}=450-600 \mathrm{~nm}, 1 \mathrm{~nm}$ intervals) obtained from purified RNCs were measured at $23{ }^{\circ} \mathrm{C}$ using a Fluorolog 3-22 fluorometer (HORIBA Jobin Yvon, Edison, NJ). 5 highest peak emission intensities $(\sim 475 \mathrm{~nm})$ were averaged and used for subsequent calculation. Ribosome release was performed by adding column buffer with final concentrations of $200 \mu \mathrm{g} / \mathrm{ml}$ RNase A and $3 \mathrm{mM}$ ATP. At the end of measurement, the concentration of polypeptide in $\mathrm{D}$ and $\mathrm{D}+\mathrm{A}$ samples was determined by ${ }^{14} \mathrm{C}$ scintillation counting using the Eq. (2). FRET efficiency was then calculated by the acceptor-dependent decrease in CFP fluorescence intensity from the Eq. (1) as described in "Results" section.

Bead binding and assay plate preparation. RNC binding to beads was carried out in $400 \mu \mathrm{l}$ binding buffer (column buffer plus $0.5 \mathrm{mM}$ DTT and $20 \mathrm{mM}$ Imidazole-HCl pH 7.2) in a Protein LoBind round-bottom polypropylene tube $2.0 \mathrm{~mL}$ (Eppendorf) using a Fisher Scientific 346 Hematology/Chemistry Mixer at room temperature. Typical conditions involved $2 \mathrm{nM}$ RNCs, $6 \mathrm{~h}$ incubation time length, and $2 \times 10^{5}$ of High Density Nickel 4 Highly Cross-linked Superfine $17 \mu \mathrm{m}$ (Agarose Bead Technologies, $17 \mu \mathrm{m}$ beads), $5 \times 10^{4}$ of HiTrap Chelating HP (GE Healthcare, $34 \mu \mathrm{m}$ beads) charged with Nickel as described elsewhere ${ }^{65}$, or $6 \times 10^{3}$ of Ni-NTA agarose (Qiagen, $100 \mu \mathrm{m}$ beads) unless otherwise stated. For high-throughput screening (HTS), bead binding was carried out with $2 \mathrm{nM}$ RNCs, and $1 \times 10^{6}$ of $17 \mu \mathrm{m}$ beads in $800 \mu$ l binding buffer over-night $(16 \mathrm{~h})$ at room temperature. Beads were then transferred to V-bottom shaped $1.5 \mathrm{~mL}$ Eppendorf tube and washed $\times 3$ with $1 \mathrm{ml}$ of binding buffer followed by centrifugation at $5,000 \times g$ at room temperature for $1 \mathrm{~min}$. $\mathrm{D}$ and $\mathrm{D}+\mathrm{A}$ beads were mixed in $50 \mathrm{~mL}$ conical tubes with $6.7 \mathrm{ml}$ of column buffer plus $0.5 \mathrm{mM}$ DTT (150 beads/ $\mu$ lat final concentration). 1,536 well (or 96/384 well for earlier experiments) glass bottom plates (Greiner Bio-One, Cat\# 783892 or Cellvis, Cat\# P96/384-1.5H-N) were used as assay plates. 600 beads, 500 beads, or 1,000 beads were dispensed into each well of 1,536 well, 384 well, or 96 well plates, respectively, by Multidrop Combi Reagent Dispenser (Thermo Scientific) and allowed to settle to well bottom by gravity for $30 \mathrm{~min}$. Final assay volumes were adjusted to $7.5 \mu \mathrm{l}, 50 \mu \mathrm{l}$, or $200 \mu \mathrm{l}$ in each well by dispensing column buffer plus $0.5 \mathrm{mM}$ DTT into 1,536 well, 384 well, or 96 well plates, respectively, prior to dispensing bead solution. Where indicated, nascent chains were released from ribosomes by addition of $200 \mu \mathrm{g} / \mathrm{ml}$ RNase A and $3 \mathrm{mM}$ ATP at final concentrations. RNC surface density was calculated using number of total protein molecules bound (derived from ${ }^{14} \mathrm{C}$ counting using the Eq. (2) and total surface area of beads as described in "Results" section. Bead binding for free nascent chain was performed by preincubating RNCs incubated with RNase A $(0.2 \mathrm{mg} / \mathrm{ml})$ for $15 \mathrm{~min}$ at room temperature prior to incubation with beads.

HTS. 50,240 small molecule compounds from a diversified library (ChemDiv, inc.) were tested in this study. $7.5 \mathrm{nl}$ of $10 \mathrm{mM}$ compounds in 100\% DMSO were dispensed into each well of 1,536 well plates (Greiner BioOne, Cat\# 783892) by Echo 550 Liquid Handler (Beckman Coulter). $3.5 \mu$ l of column buffer plus 0.5 mM DTT was then mixed with the compounds each well followed by centrifugation at $500 \times g$ at room temperature for $1 \mathrm{~min} .4 \mu \mathrm{l}$ of bead solution (150 beads/ $\mu$ l) was dispensed into each well of assay plates by Multidrop Combi Reagent Dispenser (Thermo Scientific). Each assay plate includes the vehicle control wells (0.1\% DMSO) as showing in Supplementary Figure S12. 6-point dose-response assays (5-40 $\mu \mathrm{M}$ compound, 0.4\% DMSO) were performed for primary hit compounds in the same procedures as the HTS.

Imaging and analysis. Beads were imaged in column buffer plus $0.5 \mathrm{mM} \mathrm{DTT}$ at $25^{\circ} \mathrm{C}$ for all experiments. Three microscopes were tested in developing this protocol as described below; In Cell Analyzer 2200 software version 4 or 6 (GE Healthcare) [excitation wavelength, $438 \mathrm{~nm}(426-450 \mathrm{~nm})$, emission wavelength, $475 \mathrm{~nm}$ (463-487 nm), $20 \times 0.75 \mathrm{NA}$ objective, $0.325 \times 0.325 \mathrm{~nm}^{2} /$ pixel, 1 or $0.5 \mathrm{~s}$ exposure, 1 , 4 , or 9 images per well]; Nikon Ti-E eclipse microscope with Lumencor Sola Light Engine and Andor Zyla Camera [excitation wavelength, $436 \mathrm{~nm}$ (426-446 nm), emission wavelength, $480 \mathrm{~nm}$ (460-500 nm), 20×0.75NA objective, $1 \mathrm{~s}$ exposure]; and Olympus IX71 high resolution widefield inverted microscope and features an LED transmitted light source for differential interference contrast [excitation wavelength, $430 \mathrm{~nm}$ (418-442 nm), emission wavelength, $470 \mathrm{~nm}(458-482 \mathrm{~nm}), 3 \mathrm{~s}$ exposure]. While all the three microscopes can be used to detect fluorescence signal of beads from images, the GE In Cell System was superior for HTS. For GE In Cell Analyzer, flat-field correction 
was applied to minimize well-to-well and in-well variations. Note that flat-field corrections between instrument software version 4 and 6 are not identical due to different algorithms of software, and provide substantial differences in the signal intensity (fluorescence units). This did not have effect FRET measurements as showing in Supplementary Figure S6. The central focal plane of the image was set to the distance of bead radius from the bottom of the well. HTS was carried out in 1,536 well plate format ( 1 image/well, $0.5 \mathrm{~s}$ exposure). Earlier experiments were carried out in 96 or 384 well plates ( 9 images/well, $1.0 \mathrm{~s}$ exposure). Valid bead images were selected using In Cell Developer Toolbox software v1.9 (GE Healthcare), with image segmentation method as described below. Unfocused beads and artifact objects were eliminated by applying restrictions of diameter (16-23 $\mu \mathrm{m}$ for $17 \mu \mathrm{m}$ beads), circularity ( $>0.93)$, and threshold pixel intensity. Mean fluorescent intensity of selected beads was quantitated per unit area, and data was analyzed using excel and/or prism software. FRET efficiency was calculated by the Eq. (1) as above. For earlier experiments, the $Z$ ' factor ${ }^{53}$ was calculated from the following equation:

$$
\mathrm{Z}^{\prime}=1-3 \times\left(\mathrm{SD}_{\mathrm{P}}+\mathrm{SD}_{\mathrm{N}}\right) /\left(\mathrm{Mean}_{\mathrm{P}}-\mathrm{Mean}_{\mathrm{N}}\right)
$$

where $\mathrm{SD}_{\mathrm{P}}$ and $\mathrm{SD}_{\mathrm{N}}$ are standard deviation of positive and negative controls, respectively. Mean $\operatorname{and~Mean~}_{\mathrm{N}} \operatorname{are}$ mean of positive and negative controls, respectively. For practical purposes, positive control is $\Delta \mathrm{RI}$ and negative control is wild-type.

Immunoblotting. Human embryonic kidney (HEK) 293 cells (ATCC, Cat\# CRL-1573) were grown at $37^{\circ} \mathrm{C}$ under $5 \% \mathrm{CO}_{2}$ in Dulbecco's modified Eagle's medium (Invitrogen) supplemented with $10 \%$ fetal calf serum (Thermo Scientific) and penicillin/streptomycin (Invitrogen). $5 \times 10^{5}$ cells were seeded in six-well plates and transfected one day later with equal amounts $(2.5 \mu \mathrm{g})$ of pcDNA3-CFTR vector containing wild-type or A455E as indicated. Cells were treated with compounds (final 1\% DMSO) $24 \mathrm{~h}$ after transfection and harvested $24 \mathrm{~h}$ after compound treatment by lysis for $20 \mathrm{~min}$ in $600 \mu \mathrm{l}$ of ice-cold RIPA buffer $(20 \mathrm{mM} \mathrm{HEPES}-\mathrm{NaOH} / \mathrm{pH} 7.5$, $150 \mathrm{mM} \mathrm{NaCl}, 1 \mathrm{mM}$ EDTA, $1 \%$ Triton X-100, 0.1\% SDS, 0.5\% sodium deoxycholate) containing cOmplete Protease Inhibitor Cocktail (Roche Applied Science). Cell lysate was separated by SDS-PAGE, transferred to PVDF membrane (Millipore), and immunoblotted using the following primary antibodies: (1) mouse anti-CFTR antibody M3A7 (Millipore, Cat\# 05-593, Lot\# 2652963, 1:2,000 dilution), and (2) rabbit anti- $\beta$-actin (Santa Cruz Biotech., Cat\# sc-47778, Lot\# B1914, 1:2,000 dilution) and secondary antibodies: (3) goat anti-mouse IgG $(\mathrm{H}+\mathrm{L}$ )-HRP conjugate (Bio-Rad, Cat\# 1706516, 1:5,000 dilution), or (4) goat anti-rabbit IgG-HRP (Santa Cruz Biotech., Cat\# sc-2030, 1:5,000 dilution). Blots were imaged using the ChemiDoc XRS + System (Bio-Rad) and analyzed using accompanying image analysis software.

$\left[{ }^{35} \mathrm{~S}\right]$-methionine pulse-chase labeling. HEK 293 cells were grown at $37{ }^{\circ} \mathrm{C}$ under $5 \% \mathrm{CO}_{2}$ in $\mathrm{Dul}-$ becco's modified Eagle's medium (Invitrogen) supplemented with $10 \%$ fetal calf serum (Thermo Scientific) and penicillin/streptomycin (Invitrogen). $5 \times 10^{5}$ cells were seeded in six-well plates and transfected one day later with equal amounts $(2.5 \mu \mathrm{g})$ of pcDNA3-NBD1 vector containing wild-type or A455E as indicated. Cells were treated with or without compound treatment (final 1\% DMSO) $24 \mathrm{~h}$ after transfection, incubated for $1 \mathrm{~h}$ in cysteine and methionine-free medium with the compound $1 \mathrm{~h}$ after compound treatment, pulsed labeled with $30 \mu \mathrm{Ci}$ Trans ${ }^{35} \mathrm{~S}$-label (MP Biomedicals)/well with the compound for $30 \mathrm{~min}$, chased with the regular media and the compound for $0-4 \mathrm{~h}$, and harvested directly at the indicated times by lysis for $20 \mathrm{~min}$ in $600 \mu \mathrm{l}$ of ice-cold RIPA buffer containing complete protease inhibitor mixture. Cell lysate was incubated with anti-CFTR 3G11 rat monoclonal antibody (Cystic Fibrosis Folding Consortium) overnight at $4{ }^{\circ} \mathrm{C}$, and then for $2 \mathrm{~h}$ at $4{ }^{\circ} \mathrm{C}$ after addition of Affi-gel Protein G (Bio-Rad). Protein G beads were washed five times with RIPA buffer and mixed with SDS-PAGE sample buffer, followed by separation on SDS-PAGE. The radio-labeled bands were imaged and analyzed with the Personal FX phosphor imager and Quantity One software (Bio-Rad).

\section{Data availability}

All data in this study are available from the corresponding author upon reasonable request.

Received: 27 June 2021; Accepted: 18 January 2022

Published online: 15 February 2022

\section{References}

1. Khushoo, A., Yang, Z., Johnson, A. E. \& Skach, W. R. Ligand-driven vectorial folding of ribosome-bound human CFTR NBD1. Mol. Cell 41, 682-692 (2011).

2. Kim, S. J. et al. Protein folding. Translational tuning optimizes nascent protein folding in cells. Science 348, 444-448 (2015).

3. Waudby, C. A., Dobson, C. M. \& Christodoulou, J. Nature and regulation of protein folding on the ribosome. Trends Biochem. Sci. 44, 914-926 (2019).

4. Kramer, G., Shiber, A. \& Bukau, B. Mechanisms of cotranslational maturation of newly synthesized proteins. Annu. Rev. Biochem. 88, 337-364 (2019).

5. Bitran, A., Jacobs, W. M., Zhai, X. \& Shakhnovich, E. Cotranslational folding allows misfolding-prone proteins to circumvent deep kinetic traps. Proc. Natl. Acad. Sci. USA 117, 1485-1495 (2020).

6. Walsh, I. M., Bowman, M. A., Soto Santarriaga, I. F., Rodriguez, A. \& Clark, P. L. Synonymous codon substitutions perturb cotranslational protein folding in vivo and impair cell fitness. Proc. Natl. Acad. Sci. USA 117, 3528-3534 (2020).

7. Stein, K. C. \& Frydman, J. The stop-and-go traffic regulating protein biogenesis: How translation kinetics controls proteostasis. J. Biol. Chem. 294, 2076-2084 (2019).

8. Liu, K., Maciuba, K. \& Kaiser, C. M. The ribosome cooperates with a chaperone to guide multi-domain protein folding. Mol. Cell 74, 310-3197 (2019).

9. Stein, K. C., Kriel, A. \& Frydman, J. Nascent polypeptide domain topology and elongation rate direct the cotranslational hierarchy of Hsp70 and TRiC/CCT. Mol. Cell 75, 1117-1130 e5 (2019). 
10. McGuffee, S. R. \& Elcock, A. H. Diffusion, crowding \& protein stability in a dynamic molecular model of the bacterial cytoplasm. PLoS Comput. Biol. 6, e1000694 (2010).

11. Elcock, A. H. Models of macromolecular crowding effects and the need for quantitative comparisons with experiment. Curr. Opin. Struct. Biol. 20, 196-206 (2010).

12. Chu, X., Suo, Z. \& Wang, J. Confinement and crowding effects on folding of a multidomain Y-family DNA polymerase. J. Chem. Theory Comput. 16, 1319-1332 (2020).

13. Sander, I. M., Chaney, J. L. \& Clark, P. L. Expanding Anfinsen's principle: contributions of synonymous codon selection to rational protein design. J. Am. Chem. Soc. 136, 858-861 (2014).

14. Ugrinov, K. G. \& Clark, P. L. Cotranslational folding increases GFP folding yield. Biophys. J. 98, 1312-1320 (2010).

15. Vembar, S. S. \& Brodsky, J. L. One step at a time: endoplasmic reticulum-associated degradation. Nat. Rev. Mol. Cell Biol. 9, 944-957 (2008).

16. Carvalho, P., Goder, V. \& Rapoport, T. A. Distinct ubiquitin-ligase complexes define convergent pathways for the degradation of ER proteins. Cell 126, 361-373 (2006).

17. Denic, V., Quan, E. M. \& Weissman, J. S. A luminal surveillance complex that selects misfolded glycoproteins for ER-associated degradation. Cell 126, 349-359 (2006).

18. Brodsky, J. L. \& Skach, W. R. Protein folding and quality control in the endoplasmic reticulum: Recent lessons from yeast and mammalian cell systems. Curr. Opin. Cell Biol. 23, 464-475 (2011).

19. Frizzell, R. A. \& Hanrahan, J. W. Physiology of epithelial chloride and fluid secretion. Cold Spring Harb. Perspect. Med. 2, a009563 (2012).

20. Protasevich, I. et al. Thermal unfolding studies show the disease causing F508del mutation in CFTR thermodynamically destabilizes nucleotide-binding domain 1. Protein Sci. 19, 1917-1931 (2010).

21. Thibodeau, P. H. et al. The cystic fibrosis-causing mutation deltaF508 affects multiple steps in cystic fibrosis transmembrane conductance regulator biogenesis. J. Biol. Chem. 285, 35825-35835 (2010).

22. Rabeh, W. M. et al. Correction of both NBD1 energetics and domain interface is required to restore DeltaF508 CFTR folding and function. Cell 148, 150-163 (2012).

23. Mendoza, J. L. et al. Requirements for efficient correction of DeltaF508 CFTR revealed by analyses of evolved sequences. Cell 148, 164-174 (2012).

24. Shishido, H., Yoon, J. S., Yang, Z. \& Skach, W. R. CFTR trafficking mutations disrupt cotranslational protein folding by targeting biosynthetic intermediates. Nat. Commun. 11, 4258 (2020).

25. Marinko, J. T. et al. Folding and misfolding of human membrane proteins in health and disease: From single molecules to cellular proteostasis. Chem. Rev. 119, 5537-5606 (2019).

26. Alagramam, K. N. et al. A small molecule mitigates hearing loss in a mouse model of Usher syndrome III. Nat. Chem. Biol. 12, 444-451 (2016).

27. Conn, P. M., Smith, E., Hodder, P., Janovick, J. A. \& Smithson, D. C. High-throughput screen for pharmacoperones of the vasopressin type 2 receptor. J. Biomol. Screen. 18, 930-937 (2013).

28. Molinski, S. et al. Functional rescue of F508del-CFTR using small molecule correctors. Front. Pharmacol. 3, 160 (2012).

29. Pedemonte, N. \& Galietta, L. J. Pharmacological correctors of mutant CFTR mistrafficking. Front. Pharmacol. 3, 175 (2012).

30. Van Goor, F. et al. Rescue of DeltaF508-CFTR trafficking and gating in human cystic fibrosis airway primary cultures by small molecules. Am. J. Physiol. Lung Cell Mol. Physiol. 290, L1117-L1130 (2006).

31. Pedemonte, N. et al. Small-molecule correctors of defective DeltaF508-CFTR cellular processing identified by high-throughput screening. J. Clin. Invest. 115, 2564-2571 (2005).

32. Sampson, H. M. et al. Identification of a NBD1-binding pharmacological chaperone that corrects the trafficking defect of F508delCFTR. Chem. Biol. 18, 231-242 (2011).

33. Phuan, P. W. et al. DeltaF508-CFTR modulator screen based on cell surface targeting of a chimeric nucleotide binding domain 1 reporter. SLAS Discov. 23, 823-831 (2018).

34. Lopes-Pacheco, M. CFTR modulators: The changing face of cystic fibrosis in the era of precision medicine. Front. Pharmacol. 10, $1662(2019)$.

35. Taylor-Cousar, J. L. et al. Tezacaftor-Ivacaftor in patients with cystic fibrosis homozygous for Phe508del. N. Engl. J. Med. 377, 2013-2023 (2017).

36. Boyle, M. P. et al. A CFTR corrector (lumacaftor) and a CFTR potentiator (ivacaftor) for treatment of patients with cystic fibrosis who have a phe508del CFTR mutation: A phase 2 randomised controlled trial. Lancet Respir. Med. 2, 527-538 (2014).

37. Middleton, P. G. et al. Elexacaftor-Tezacaftor-Ivacaftor for cystic fibrosis with a single Phe508del allele. N. Engl. J. Med. 381, 1809-1819 (2019).

38. Heijerman, H. G. M. et al. Efficacy and safety of the elexacaftor plus tezacaftor plus ivacaftor combination regimen in people with cystic fibrosis homozygous for the F508del mutation: A double-blind, randomised, phase 3 trial. Lancet 394, 1940-1948 (2019).

39. Bose, S. J. et al. Towards next generation therapies for cystic fibrosis: Folding, function and pharmacology of CFTR. J. Cyst. Fibros 19(Suppl 1), S25-S32 (2020).

40. Farinha, C. M. et al. Increased efficacy of VX-809 in different cellular systems results from an early stabilization effect of F508delCFTR. Pharmacol. Res. Perspect. 3, e00152 (2015).

41. Lopes-Pacheco, M. CFTR modulators: Shedding light on precision medicine for cystic fibrosis. Front. Pharmacol. 7, 275 (2016).

42. Okiyoneda, T. et al. Mechanism-based corrector combination restores DeltaF508-CFTR folding and function. Nat. Chem. Biol. 9, 444-454 (2013).

43. Johnson, A. E. The co-translational folding and interactions of nascent protein chains: A new approach using fluorescence resonance energy transfer. FEBS Lett. 579, 916-920 (2005).

44. Kelkar, D. A., Khushoo, A., Yang, Z. \& Skach, W. R. Kinetic analysis of ribosome-bound fluorescent proteins reveals an early, stable, cotranslational folding intermediate. J. Biol. Chem. 287, 2568-2578 (2012).

45. Woolhead, C. A., McCormick, P. J. \& Johnson, A. E. Nascent membrane and secretory proteins differ in FRET-detected folding far inside the ribosome and in their exposure to ribosomal proteins. Cell 116, 725-736 (2004).

46. Sosnay, P. R. et al. Defining the disease liability of variants in the cystic fibrosis transmembrane conductance regulator gene. Nat. Genet. 45, 1160-1167 (2013).

47. Estacio, S. G., Martiniano, H. F. \& Faisca, P. F. Thermal unfolding simulations of NBD1 domain variants reveal structural motifs associated with the impaired folding of F508del-CFTR. Mol. Biosyst. 12, 2834-2848 (2016).

48. Hoelen, H. et al. The primary folding defect and rescue of DeltaF508 CFTR emerge during translation of the mutant domain. PLoS ONE 5, e15458 (2010).

49. Veit, G. et al. Structure-guided combination therapy to potently improve the function of mutant CFTRs. Nat. Med. 24, 1732-1742 (2018).

50. Aleksandrov, A. A. et al. Regulatory insertion removal restores maturation, stability and function of DeltaF508 CFTR. J. Mol. Biol. 401, 194-210 (2010).

51. Atwell, S. et al. Structures of a minimal human CFTR first nucleotide-binding domain as a monomer, head-to-tail homodimer, and pathogenic mutant. Protein. Eng. Des. Sel. 23, 375-384 (2010). 
52. Wang, C. et al. Integrated biophysical studies implicate partial unfolding of NBD1 of CFTR in the molecular pathogenesis of F508del cystic fibrosis. Protein. Sci. 19, 1932-1947 (2010).

53. Zhang, J. H., Chung, T. D. \& Oldenburg, K. R. A simple statistical parameter for use in evaluation and validation of high throughput screening assays. J. Biomol. Screen 4, 67-73 (1999).

54. He, L. et al. Restoration of NBD1 thermal stability is necessary and sufficient to correct F508 CFTR folding and assembly. J. Mol. Biol. 427, 106-120 (2015).

55. Sigoillot, M. et al. Domain-interface dynamics of CFTR revealed by stabilizing nanobodies. Nat. Commun. 10, 2636 (2019).

56. Tosatto, L. et al. Single-molecule FRET studies on alpha-synuclein oligomerization of Parkinson's disease genetically related mutants. Sci. Rep. 5, 16696 (2015).

57. Nissley, D. A. \& O'Brien, E. P. Structural origins of FRET-observed nascent chain compaction on the ribosome. J. Phys. Chem. B 122, 9927-9937 (2018).

58. Wang, S. et al. Potassium channel selectivity filter dynamics revealed by single-molecule FRET. Nat. Chem. Biol. 15, 377-383 (2019).

59. Bertolin, G. et al. A FRET biosensor reveals spatiotemporal activation and functions of aurora kinase A in living cells. Nat Commun 7, 12674 (2016).

60. Chung, C. I. et al. Intrabody-based FRET probe to visualize endogenous histone acetylation. Sci. Rep. 9, 10188 (2019).

61. Schuler, B., Soranno, A., Hofmann, H. \& Nettels, D. Single-molecule FRET spectroscopy and the polymer physics of unfolded and intrinsically disordered proteins. Annu. Rev. Biophys. 45, 207-231 (2016).

62. Devanarayan, V. et al. Glossary of quantitative biology terms. In Assay Guidance Manual (eds. Markossian, S. et al.) (Bethesda (MD), 2004)

63. Matsumura, Y., Rooney, L. \& Skach, W. R. In vitro methods for CFTR biogenesis. Methods Mol. Biol. 741, 233-253 (2011).

64. Gubbens, J., Kim, S. J., Yang, Z., Johnson, A. E. \& Skach, W. R. In vitro incorporation of nonnatural amino acids into protein using tRNA(Cys)-derived opal, ochre, and amber suppressor tRNAs. RNA 16, 1660-1672 (2010).

65. Piston, D. W. \& Rizzo, M. A. FRET by fluorescence polarization microscopy. Methods Cell Biol. 85, 415-430 (2008).

\section{Acknowledgements}

We thank members of the CFFT Lab for helpful discussions. This research was funded by the Cystic Fibrosis Foundation, NIH Grants R01GM53457 and R01DK51818 (to W.R.S.), Cystic Fibrosis Foundation Therapeutics Inc. grant SKACH05X0 (to W.R.S.), and American Lung Association Grant RT-269559-N (to H.S.).

\section{Author contributions}

H.S. designed and executed the series of experiments, analyzed results, and wrote the manuscript. J.S.Y. assisted in pulse-chase experiments and writing the manuscript. W.R.S. conceived the project, designed experiments, and assisted in writing the manuscript.

\section{Competing interests}

H.S. is employed at Generate Biomedicines, Inc., though at time of studying and writing his employment was at the Cystic Fibrosis Foundation. H.S. is a member of the board of directors of Inter Action Corp. (Japan). J.Y.S. and W.R.S. are employees of the Cystic Fibrosis Foundation, which is involved in and funding independent programs for CF drug development including CFTR modulators that improve CFTR folding. J.Y.S. and W.R.S. declare no personal competing financial or non-financial interests with pharmaceutical companies, although CFF may receive royalties on marketed products that it has financially supported.

\section{Additional information}

Supplementary Information The online version contains supplementary material available at https://doi.org/ 10.1038/s41598-022-06456-5.

Correspondence and requests for materials should be addressed to W.R.S.

Reprints and permissions information is available at www.nature.com/reprints.

Publisher's note Springer Nature remains neutral with regard to jurisdictional claims in published maps and institutional affiliations.

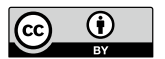

Open Access This article is licensed under a Creative Commons Attribution 4.0 International License, which permits use, sharing, adaptation, distribution and reproduction in any medium or format, as long as you give appropriate credit to the original author(s) and the source, provide a link to the Creative Commons licence, and indicate if changes were made. The images or other third party material in this article are included in the article's Creative Commons licence, unless indicated otherwise in a credit line to the material. If material is not included in the article's Creative Commons licence and your intended use is not permitted by statutory regulation or exceeds the permitted use, you will need to obtain permission directly from the copyright holder. To view a copy of this licence, visit http://creativecommons.org/licenses/by/4.0/.

(c) The Author(s) 2022 